\title{
Nonlinear Dynamic Inversion Baseline Control Law: Architecture and Performance Predictions
}

\author{
Christopher J. Miller ${ }^{1}$ \\ NASA Dryden Flight Research Center, Edwards, California, 93523
}

\begin{abstract}
A model reference dynamic inversion control law has been developed to provide a baseline control law for research into adaptive elements and other advanced flight control law components. This controller has been implemented and tested in a hardware-in-the-loop simulation; the simulation results show excellent handling qualities throughout the limited flight envelope. A simple angular momentum formulation was chosen because it can be included in the stability proofs for many basic adaptive theories, such as model reference adaptive control. Many design choices and implementation details reflect the requirements placed on the system by the nonlinear flight environment and the desire to keep the system as basic as possible to simplify the addition of the adaptive elements. Those design choices are explained, along with their predicted impact on the handling qualities.
\end{abstract}

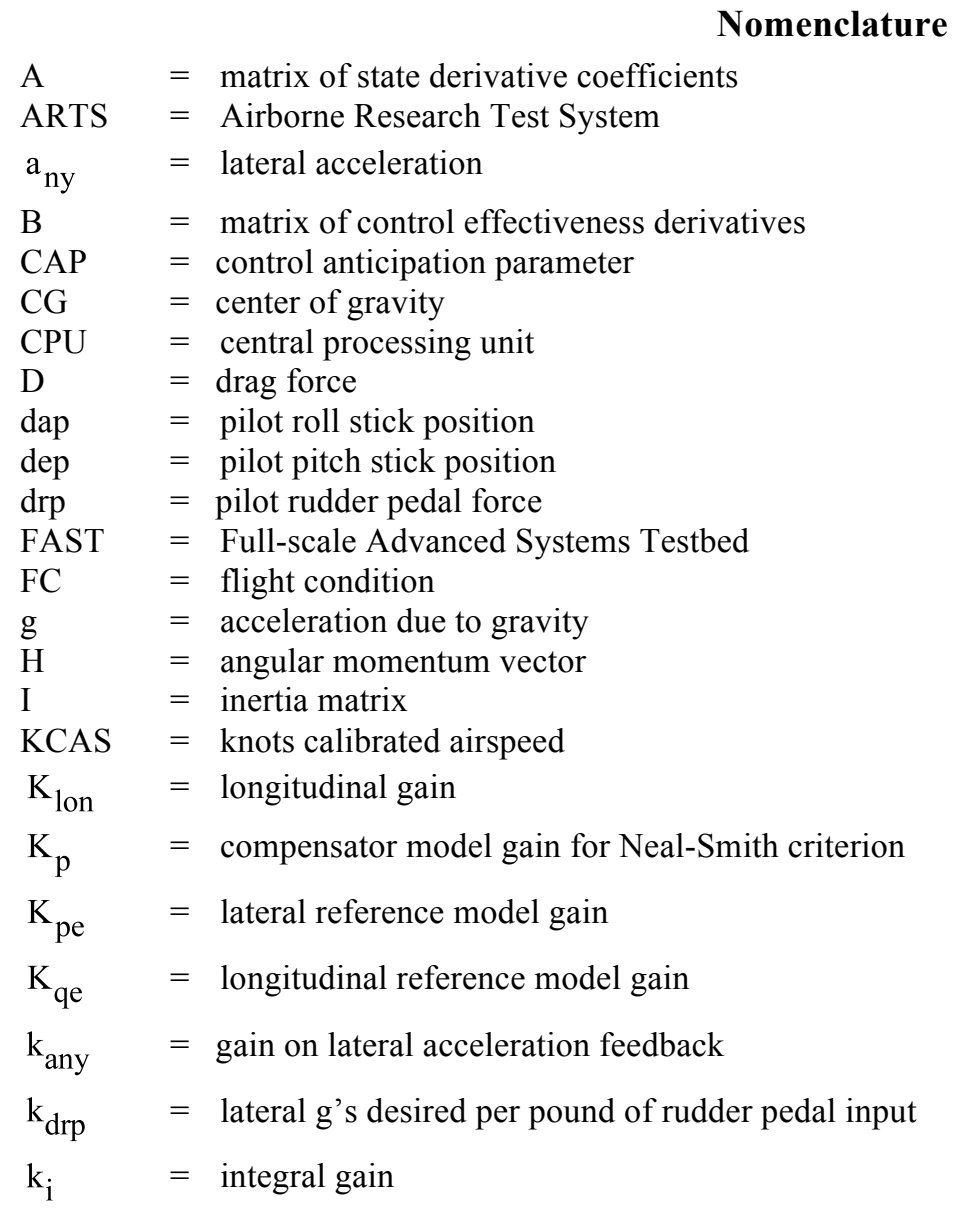

\footnotetext{
${ }^{1}$ Research Engineer, Flight Controls and Dynamics, P.O. Box 273, MS 4840D, Edwards, California, 93523, AIAA Member.
} 


\begin{tabular}{|c|c|}
\hline $\mathrm{k}_{\mathrm{p}}$ & $=$ proportional gain \\
\hline $\mathrm{L}$ & $=$ rolling moment \\
\hline LEF & $=$ leading edge flap \\
\hline $\mathrm{L}_{\alpha}$ & $=$ lift with respect to angle of attack \\
\hline M & $=$ pitching moment \\
\hline MRAC & $=$ model reference adaptive control \\
\hline MUAD & $=$ maximum unnoticeable added dynamics \\
\hline $\mathrm{m}$ & $=$ mass \\
\hline $\mathrm{N}$ & $=$ yawing moment \\
\hline NDI & $=$ nonlinear dynamic inversion \\
\hline PI & $=$ proportional integral controller \\
\hline PIO & $=$ pilot-induced oscillation \\
\hline $\mathrm{p}$ & $=$ roll rate \\
\hline q & $=$ pitch rate \\
\hline $\mathrm{q}_{\mathrm{c}}$ & $=$ impact pressure \\
\hline RAM & $=$ random access memory \\
\hline RFCS & $=$ Research Flight Control System \\
\hline $\mathrm{r}$ & $=$ yaw rate \\
\hline $\mathrm{S}$ & $=$ wing planform area \\
\hline s & $=$ Laplace operator \\
\hline TEF & $=$ trailing edge flap \\
\hline $\mathrm{u}$ & $=$ system input \\
\hline V & $=$ true airspeed \\
\hline $\mathrm{w}_{\mathrm{p}}$ & $=$ control weighting matrix \\
\hline $\mathrm{X}$ & $=$ axial force \\
\hline Y & $=$ side force \\
\hline $\mathrm{Z}$ & $=$ vertical force \\
\hline$\alpha$ & $=$ angle of attack \\
\hline$\beta$ & $=$ angle of sideslip \\
\hline$\varphi$ & $=$ roll angle \\
\hline$\theta$ & $=$ pitch angle \\
\hline$\tau_{\mathrm{p} 1}$ & $=$ zero for the Neal-Smith compensator model \\
\hline$\tau_{\mathrm{p} 2}$ & $=$ pole for the Neal-Smith compensator model \\
\hline$\omega$ & $=$ angular rate vector $[\mathrm{p} \mathrm{q} \mathrm{r}]$ \\
\hline$\omega_{\text {dist }}$ & $=$ disturbance angular rate \\
\hline$\omega_{\mathrm{fbk}}$ & $=$ angular rate feedback \\
\hline$\omega_{\mathrm{p}}$ & $=$ inverse of the roll mode time constant $[1 / \mathrm{sec}]$ \\
\hline$\omega_{\mathrm{r}}$ & $=$ inverse of the yaw axis time constant $[1 / \mathrm{sec}]$ \\
\hline$\omega_{\mathrm{sp}}$ & $=$ short period natural frequency \\
\hline$\xi_{\mathrm{sp}}$ & $=$ short period damping ratio \\
\hline
\end{tabular}

\section{Superscripts}

$\begin{array}{ll}\rightarrow & =\text { vector quantity } \\ \mathrm{e} & =\text { inertial reference frame } \\ \mathrm{b} & =\text { body reference frame }\end{array}$




\begin{tabular}{|c|c|}
\hline \multicolumn{2}{|c|}{ Subscripts } \\
\hline c & $=$ command parameter \\
\hline filt & $=$ filtered quantity \\
\hline ref & $=$ reference model parameter \\
\hline $\mathrm{s}$ & $=$ stability axis parameter \\
\hline trim & $=$ trim-related parameter \\
\hline
\end{tabular}

\section{Introduction}

$\mathrm{D}$

YNAMIC inversion as a control architecture is not new and has gained such popularity that it is being applied to new production vehicles. ${ }^{1,2}$ Thus it is not the intent of this paper to prove the viability of this type of control scheme, but rather to discuss the implementation details for a simple dynamic inversion control law. This architecture is designed specifically to be a baseline controller upon which advanced control elements can be easily added. This architecture will enable further control research into adaptive controls ${ }^{3-10}$ and the control of flexible structures. ${ }^{11,}{ }^{12}$ This baseline control law is a first step toward building a working environment in which design changes and new research objectives can be quickly brought to flight and their real behavior ascertained. The choice of dynamic inversion was driven by the intuitive architecture, explicit model-following behavior, the ability to be used to introduce fundamental-level simulated failures within the aerodynamic model for testing the performance of advanced control elements, and by the fact that dynamic inversion can be included in the stability proofs for many advanced control schemes. ${ }^{3}$

The focus of this paper is to describe the control law and provide insight into the design choices that facilitated the inclusion of adaptive elements ${ }^{3,4}$ and any that had an effect on predicted handling qualities. Each component is described in detail, and the fundamentals explained. Simplicity of formulation was an important design goal for this control scheme, but areas in which additional complexity could provide some benefit will be highlighted. In addition to describing the control architecture, this paper discusses the predicted handling qualities, and stability margins, thus providing a reference point for comparisons to flight-test results. ${ }^{13}$

\section{Background}

The control law that is the subject of this report has been designed to be an available baseline control law for the Full-scale Advanced Systems Testbed (FAST) platform. The FAST platform is fundamentally a single-seat F/A-18 airplane (McDonnell Douglas, now The Boeing Company, Chicago, Illinois), as shown in Fig. 1 and Fig. 2. Substantial research instrumentation (structural, air data, and inertial) was installed on the airplane for the Active Aeroelastic Wing (AAW) program. ${ }^{14}$ The robust nature of the testbed (structural, spin and recovery characteristics, and production control laws), along with the research instrumentation enable flight-testing of novel control laws with minimal validation testing requirements for a piloted flight vehicle.

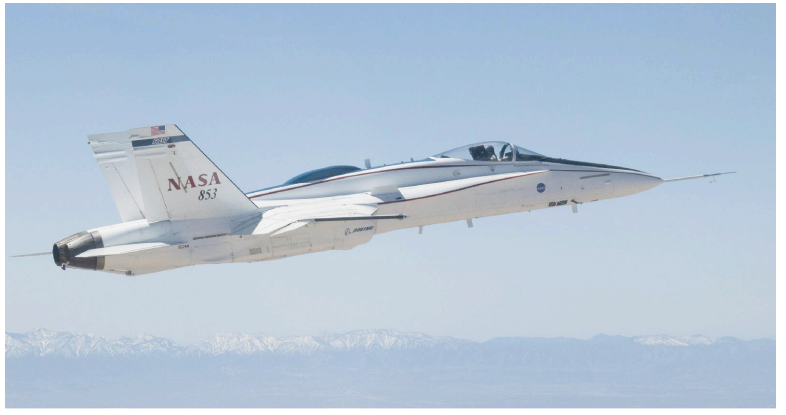

Figure 1. The Full-scale Advanced Systems Testbed F/A-18 airplane in flight.

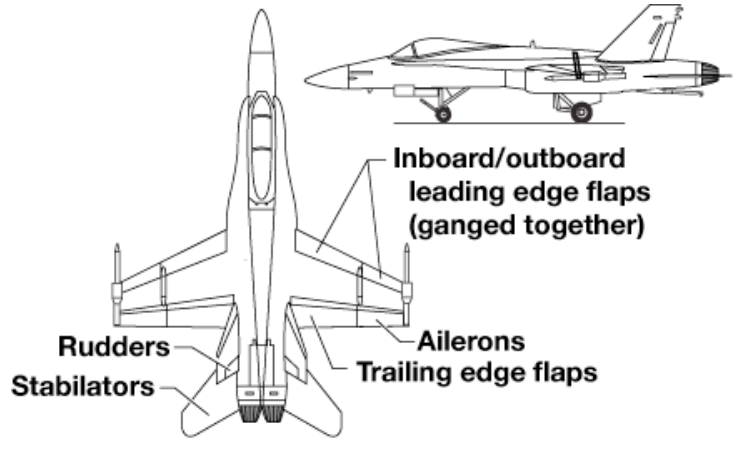

Figure 2. The control surfaces of the F/A-18 airplane. 
Figure 3 shows the control computer architecture for FAST. The system maintains the advantages of the production system and utilizes its redundancy management architecture for sensor selection and actuator signal management. The Research Flight Control System (RFCS) provides a minimal-delay, quad-redundant environment in which Ada-programmed experiments can be executed. The RFCS also performs some envelope protection for restricting those envelopes within which a given research control law can remain engaged. The Airborne Research Test System (ARTS) IV with its more capable processor and the ability to host Simulink ${ }^{\circledR}$ (The MathWorks, Inc., Natick, Massachusetts) autocode or C code provides a more flexible environment for novel control laws than does the RFCS. The ARTS, however, is only dual-redundant, and imparts one additional frame of delay to commands. This extra frame of delay translates to 0.0125 seconds for the pitch and roll axes and 0.025 seconds for the yaw axis.

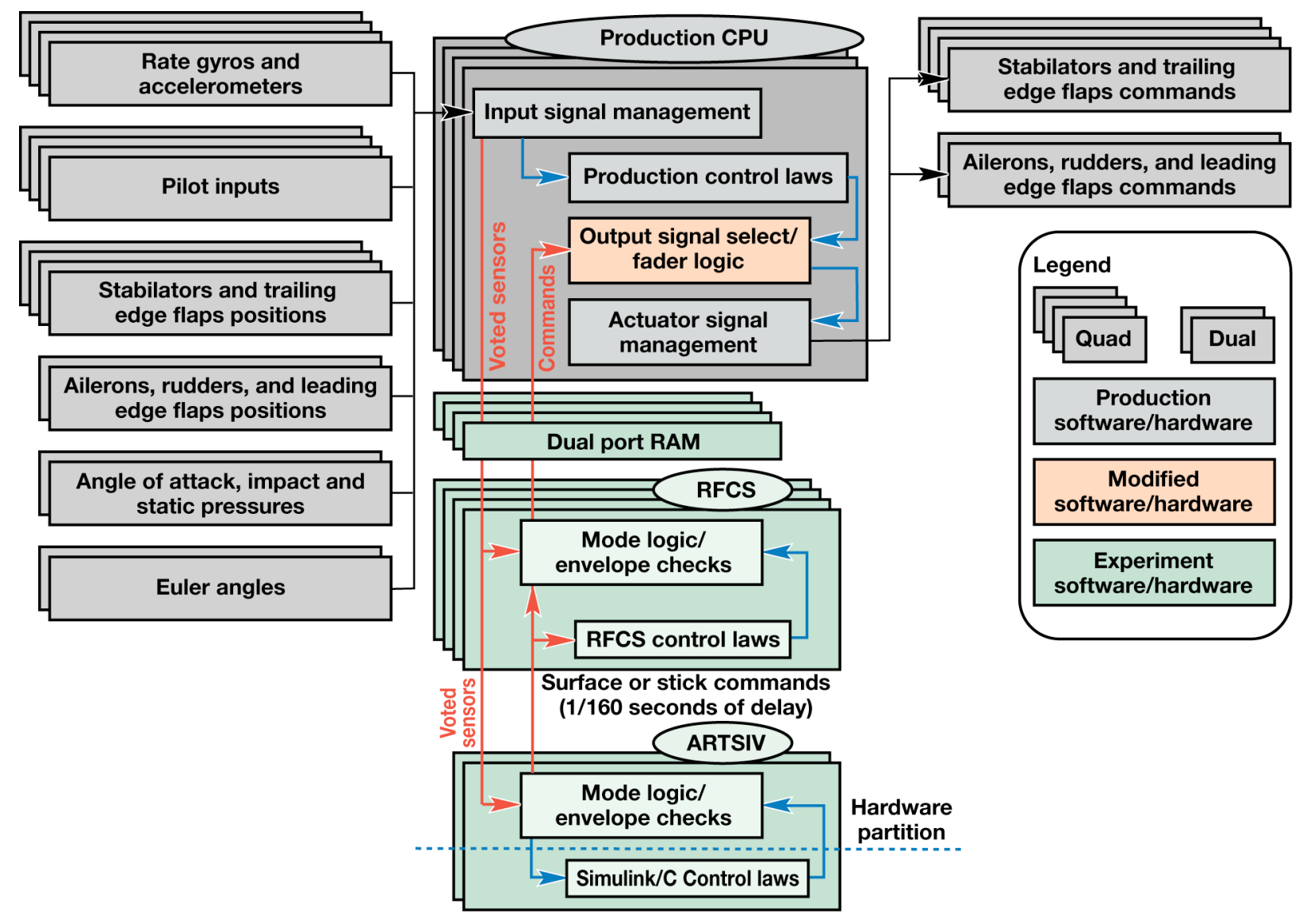

Figure 3. The Full-scale Advanced Systems Testbed Research Flight Control System Airborne Research Test System control computer architecture.

In addition to the flight assets, a simulation facility provides a hardware-in-the-loop environment for design and testing of new control techniques. The facility consists of an F/A-18 test bench with flight control hardware, a full nonlinear simulation environment, and hardware ARTS units. Without this facility, the rapid design and prototyping would not be possible.

\section{Control Law Description}

The nonlinear dynamic inversion (NDI) control law considered here contains a number of distinct components (see Fig. 4), each with their own design goals and functions. At the core of the control law is the actual dynamic inversion, which computes the surface positions necessary to achieve the desired aircraft dynamics. These desired aircraft dynamics are computed from the pilot stick commands via the use of transfer-function-based reference models. The goal is to give the pilot the type of vehicle response that is desired and expected; however, as with any real system, the model cannot be expected to exactly predict the actual behavior of the vehicle. Therefore, a compensator must be added in order to provide the necessary robustness to these modeling inaccuracies. This 
compensation is accomplished by adding a proportional-plus-integral compensator that is intended to drive down the error between the desired dynamics and the actual dynamics. Structural filters are also needed to attenuate the structural vibration from the feedback sensors in order to prevent any kind of undesirable aeroservoelastic behaviors. This basic and simple architecture was chosen based on its applicability to adaptive flight control research;, 7 however, it need not be limited to adaptive control research. ${ }^{11}{ }^{12}$ Figure 5 through Fig. 8 show more detailed block diagrams broken out into the different control loops within the control law.

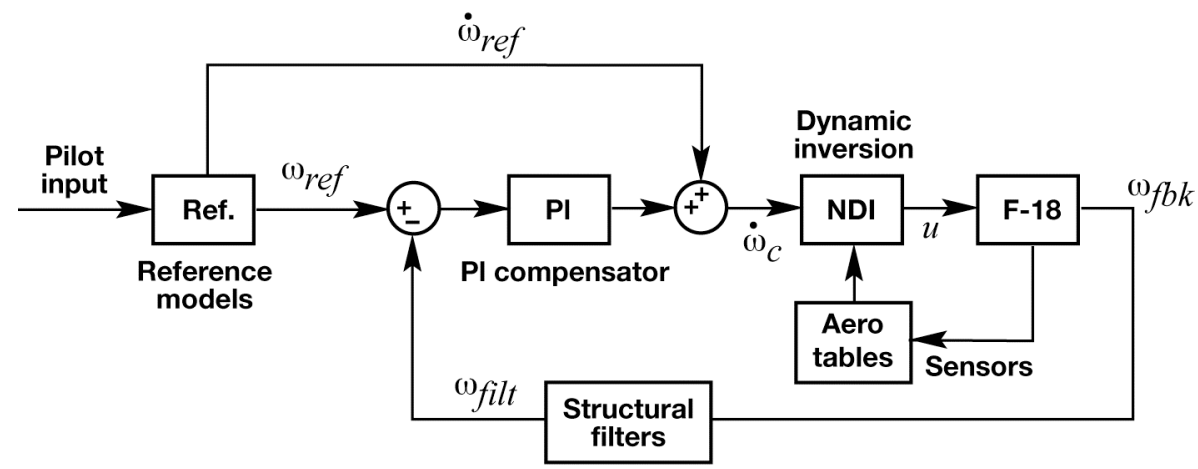

Figure 4. Block diagram of the nonlinear dynamic inversion control law.

$80 \mathrm{~Hz}$ (even $160 \mathrm{~Hz}$ frames)

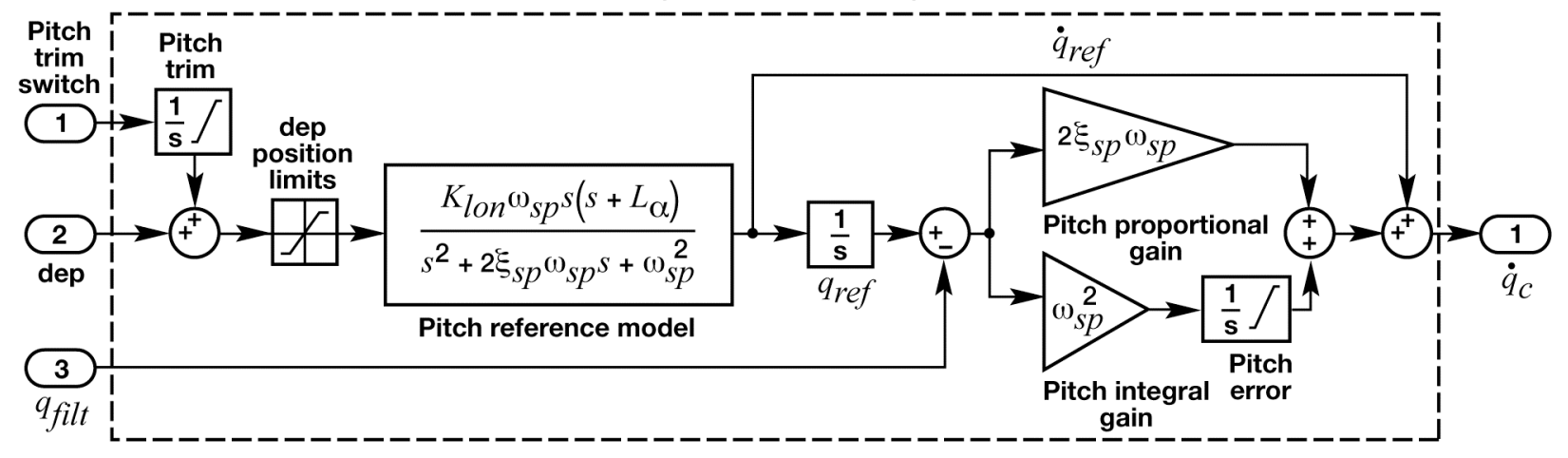

Figure 5. Block diagram of the pitch axis command loop.

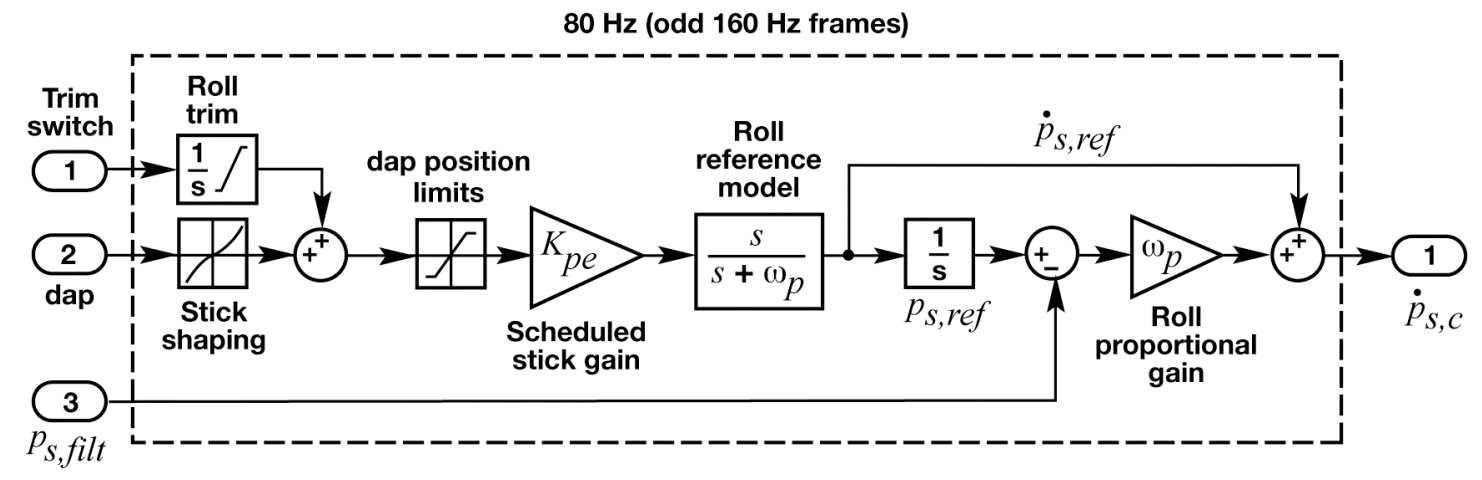

Figure 6. Block diagram of the roll axis command loop. 
$40 \mathrm{~Hz}$ (every other odd $160 \mathrm{~Hz}$ frame)

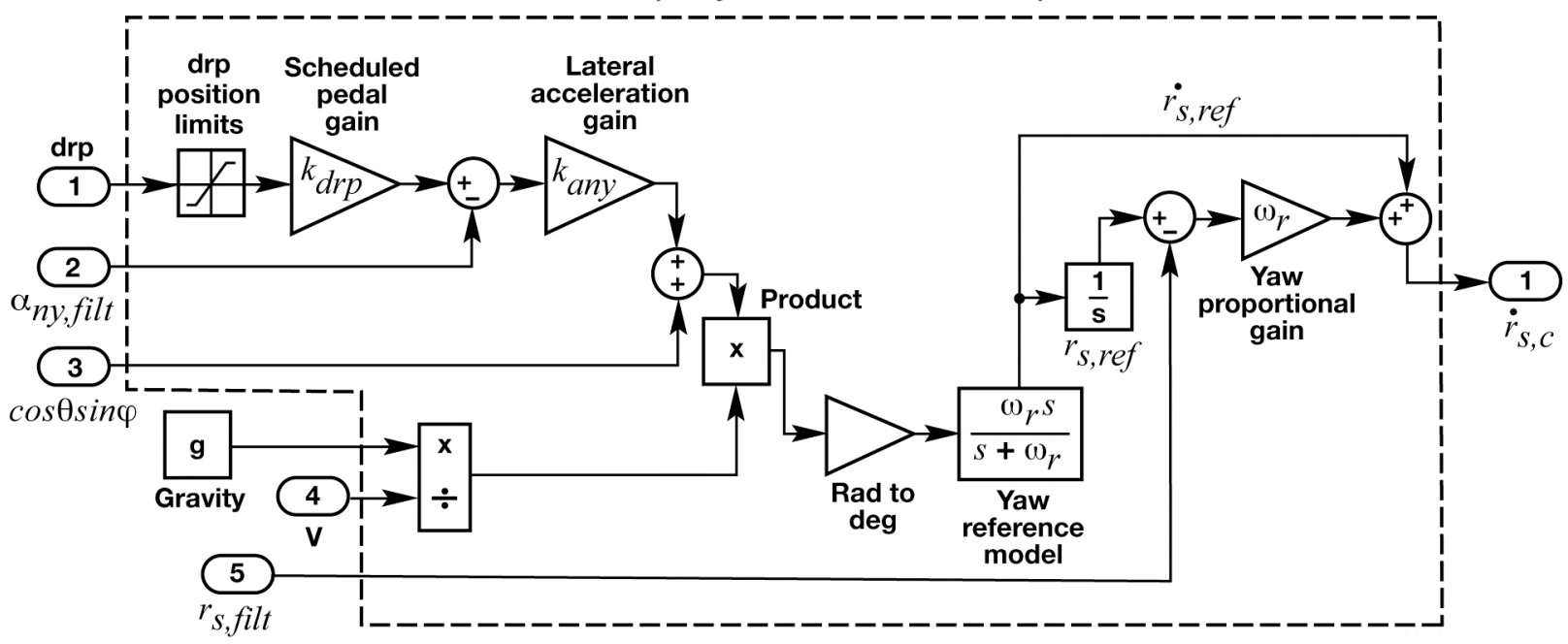

Figure 7. Block diagram of the yaw axis command loop.

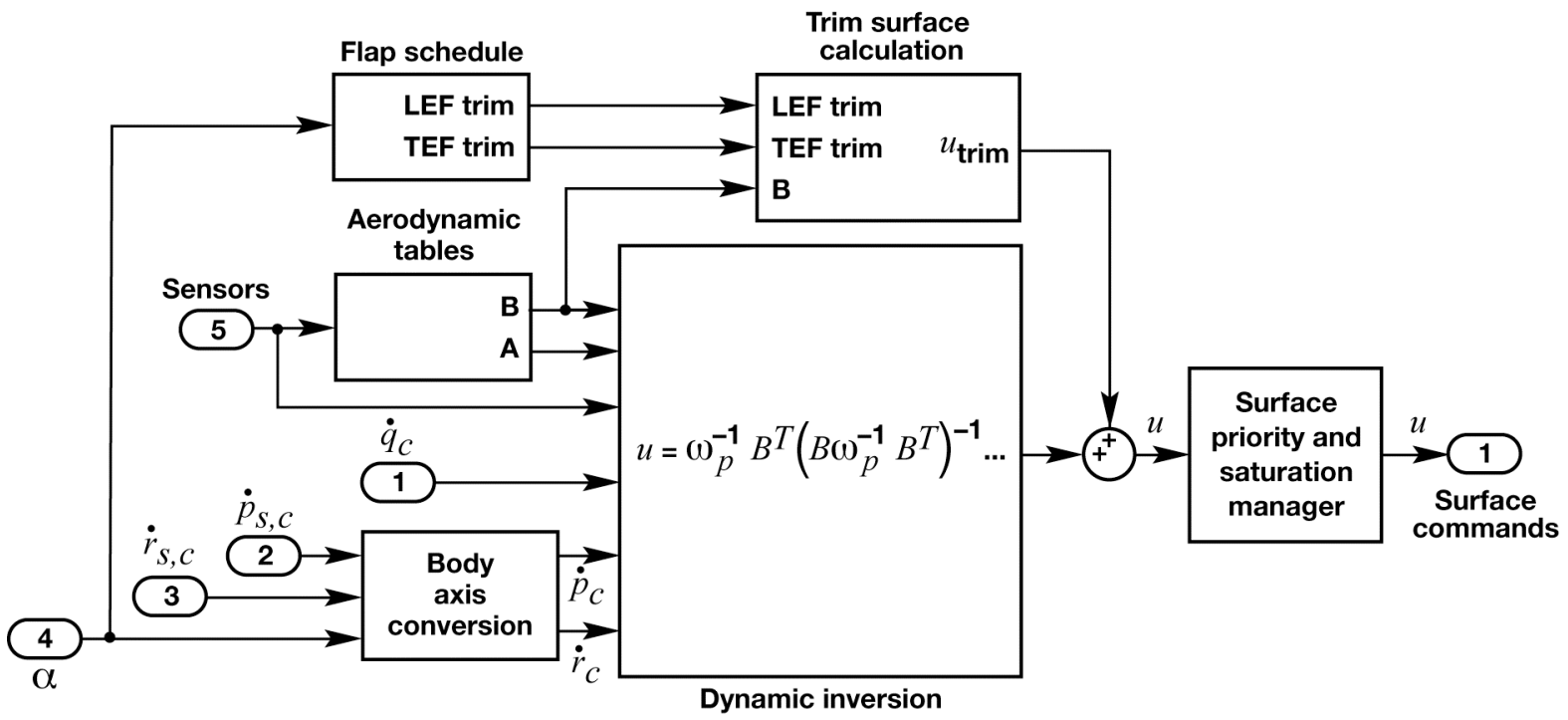

Figure 8. Block diagram of dynamic inversion.

\section{A. Feedback Linearization via Dynamic Inversion}

The dynamic inversion for this control law is based on conservation of angular momentum. Different formulations exist for dynamic inversion with differing control variables; ${ }^{2,15,16}$ however, this architecture was chosen for its straightforward applicability to model reference adaptive control (MRAC) control research. ${ }^{3,4}$ Briefly summarized, given invertible plant dynamics, the control commands needed to produce a desired response can be calculated by simply inverting the known plant dynamics. It is known from conservation of angular momentum with constant inertia that, Eqs. (1) and (2): 


$$
\begin{gathered}
\overrightarrow{\dot{\omega}^{b}}+\overrightarrow{\omega^{b}} \times I \overrightarrow{\omega^{b}}=q_{c} S A x+q_{c} S B u \\
u=w_{p}^{-1} B^{T}\left(B w_{p}^{-1} B^{T}\right)^{-1}\left[\frac{1}{q_{c} S}\left(I \overrightarrow{\dot{\omega}_{c}^{b}}+\overrightarrow{\omega^{b}} \times I \overrightarrow{\omega^{b}}\right)-A x\right]
\end{gathered}
$$

The A and B matrices in Eqs. (1) and (2) represent the effects of aerodynamics on the vehicle. Detailed descriptions of the structure, content, and derivation of these matrices can be found in Appendix A. The A matrix contains the homogenous part of the aerodynamics. The need for this matrix can be eliminated if the angular accelerations can be measured. ${ }^{17}$ These measurements, however, were not available for this design so the simulation aerodynamic model was used to calculate these effects, along with the effects of the control surfaces contained in B. The primary axial forces (drag and thrust) have been left out of the formulation because they are small moment generators when compared to the other forces and moments. Also, due to the limited envelope, shown in Fig. 9, the flexibility effects causing things such as aileron roll reversal have also been left out of the formulation. The simulation aerodynamic model breakpoint structure and table formulation is otherwise maintained, and the table look-ups are performed in real time in flight to continuously calculate both A and B. This similarity between the aerodynamic models causes concerns about the usefulness of simulation testing; these concerns are addressed through the use of a contractor-provided aerodynamic model that employs an entirely different formulation and breakpoint structure (see table 1) to provide some independent verification of the simulation results.

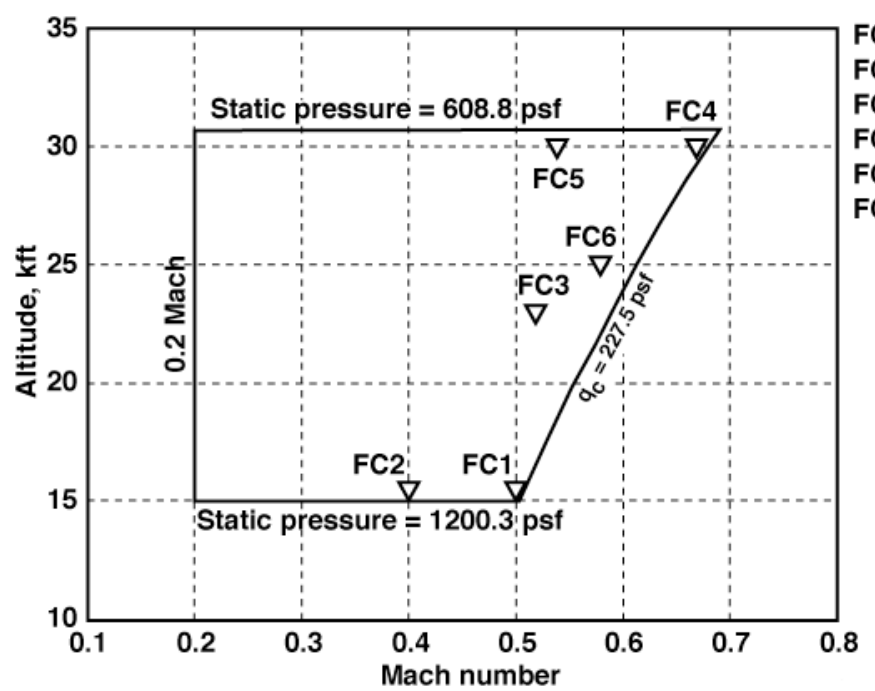

FC1: 250 KCAS, $15.5 \mathrm{kft}$

FC2: 200 KCAS, $15.5 \mathrm{kft}$ FC3: 223 KCAS, $23.0 \mathrm{kft}$ FC4: $250 \mathrm{KCAS}, 30.0 \mathrm{kft}$ FC5: $200 \mathrm{KCAS}, 30.0 \mathrm{kft}$ FC6: 240 KCAS, $25.0 \mathrm{kft}$

Figure 9. The Full-scale Advanced Systems Testbed flight envelope and flight conditions.

Table 1. List of cases tested at each flight condition.

\begin{tabular}{lccc}
\hline \hline Test case & Aerodynamic model & CG & Fuel \\
\hline 1 & Design & Aft & Nominal \\
2 & Design & Forward & Nominal \\
3 & Contractor & Nominal & Full \\
4 & Design & Nominal & Full \\
5 & Contractor & Nominal & Minimum \\
6 & Design & Nominal & Minimum \\
7 & Contractor & Nominal & Nominal \\
(Nominal) 8 & Design & Nominal & Nominal \\
\hline \hline
\end{tabular}

American Institute of Aeronautics and Astronautics 
Under most conditions, the control surface configuration of the F/A-18 airplane yields an over-determined moment command system. This fact gives rise to two design challenges: control allocation and trim solution selection.

The first challenge is addressed through the use of the weighted pseudo inverse in Eq. (2) that employs a control allocation weighting matrix, $w_{p}$. This constant diagonal matrix has been used to empirically tailor the control surface usage to mimic that of the production control law. Without this allocation weighting, the control law would utilize the surface with the highest effectiveness (largest moment derivative) in a given axis for control in that axis. This allocation scheme, while effective, results in some undesirable characteristics. For instance, it does not allow the designer to account for surface rate or position limits. The addition of the weighting matrix allows the designer to shift priority from one effector to another. This matrix was used primarily in the roll axis to shift control priority from the slower trailing edge flaps to the less effective faster ailerons, and from the stabilators to the ailerons to help offload the stabilators, which are also used heavily in pitch.

The second challenge arising from the over-determined nature of the moment control problem, that of commanding differing trim surface positions, is addressed using Eqs. (3) and (4). Close inspection reveals that Eq. (3) is equivalent to Eq. (1) provided $u_{\text {trim }}$ is in the null space of B. This addition allows the designer to specify desirable trim surface positions. For instance, leading and trailing edge flaps have been scheduled with angle of attack for this design to help keep angle of attack lower for elevated-g maneuvers.

$$
\begin{gathered}
\overrightarrow{I \dot{\omega}^{b}}+\overrightarrow{\omega^{b}} \times I \vec{\omega}=q_{c} S A x+q_{c} S B\left(u-u_{\text {trim }}\right) \\
u=w_{p}^{-1} B^{T}\left(B w_{p}^{-1} B^{T}\right)^{-1}\left[\frac{1}{q_{c} S}\left(I \overrightarrow{\dot{\omega}_{c}^{b}}+\overrightarrow{\omega^{b}} \times I \overrightarrow{\omega^{b}}\right)-A x\right]+u_{\text {trim }}
\end{gathered}
$$

\section{B. Reference Models}

Feedback linearization of the aircraft plant with dynamic inversion provides an architecture within which the vehicle will track angular acceleration commands. The standard forms for the various aircraft modes from Ref. 18 can be used to generate the desired angular accelerations from pilot commands. These standard forms can be derived from linearizations of the equations of motion in Ref. 19. For this control law, pitch stick commands generate pitch rate commands through a second order short period model, roll stick commands generate coordinated stability axis roll rate commands through a first order roll mode model, and the rudder pedal is used to generate a desired lateral acceleration.

The form of the short period transfer function implemented for the pitch reference model is the form from Ref. 18 without the time delay compensation for the higher-order dynamics, see Eq. (5). The time delay term from Ref. 18 does not apply to a reference model, as it is intended to account for higher-order vehicle dynamics; for a command path it is desirable for this term to be zero. The phugoid mode is also not included in the command reference model because it is desirable to suppress the phugoid mode.

$$
\frac{\dot{q}_{r e f}}{d e p}=\frac{K_{l o n} \omega_{s p}^{2} s\left(s+L_{\alpha}\right)}{s^{2}+2 \xi_{s p} \omega_{s p} s+\omega_{s p}^{2}}
$$

The pitch rate command architecture has a number of limitations that must be addressed. One important limitation is that it results in an undesirable unloading tendency for $360^{\circ}$ rolls. This problem arises from the fact that zero pitch stick equates to zero pitch rate command, which while inverted equates to $-1 \mathrm{~g}$. The problem is easily addressed by adding additional pitch rate commands such that zero stick equates to $1 \mathrm{~g}$ at all attitudes; however, this complicates the design such that the stability proofs for the MRAC controller became untenable. This shortcoming of the MRAC would have to be resolved for a production piloted vehicle because this behavior is very objectionable to pilots. The other limitations of the pitch rate command architecture are related to high and low dynamic pressure flight regimes. At slow speeds, aggressive pitch reference models produce large angle-of-attack rates, which can result in departure-prone configurations. At higher dynamic pressures, large pitch rates incur large normal 
accelerations quickly. Gain scheduling of the reference model parameters in Eq. (5) is one promising way to address these envelope-related limitations, as stick force per g can be specified through $\mathrm{K}_{\text {lon }}$ and $\mathrm{L}_{\alpha}$; however, this topic was not explored at this stage of the control law design.

The roll reference model takes its form from Ref. 18 as well. Equation (6) represents a simplified form with the Dutch roll and spiral modes removed. These modes can be left out of the command path because it is desirable to suppress their effects, just as it was for the phugoid mode. The other important distinction between the NDI control law roll reference model and the standard roll mode form from Ref. 18 is that the NDI reference model is implemented in the stability axis instead of the body axis for handling qualities reasons. This change in axes is driven by the fact that it is highly desirable, from a pilot's perspective, to have the aircraft maintain its load factor (angle of attack) during roll maneuvers. Rolls about the body axis have the undesirable effect of converting angle of attack to angle of sideslip. The previous discussion of the limitations of a pitch rate command system still holds. In order to completely address this undesirable unloading both the roll and the pitch aspects must be addressed; however, simply commanding stability axis rolls is sufficient for roll maneuvers that do not pass through inverted flight.

$$
\frac{\dot{p}_{\text {sref }}}{d a p}=\frac{K_{p e^{s}}}{s+\omega_{p}}
$$

Stick shaping is required in the roll axis to achieve acceptable roll handling qualities for both fine tracking and gross acquisition tasks. The goal of stick shaping is to give the pilot the ability to command large roll rates for the largest stick deflections while not making the roll axis too sensitive for small corrections. Pilot-induced oscillations (PIO) can arise from overly sensitive roll stick responses; however, high roll rates are desirable and an important performance metric. Excessive stick forces being required to generate these rates is undesirable. The stick shaping implemented for this control law is represented in Fig. 10.

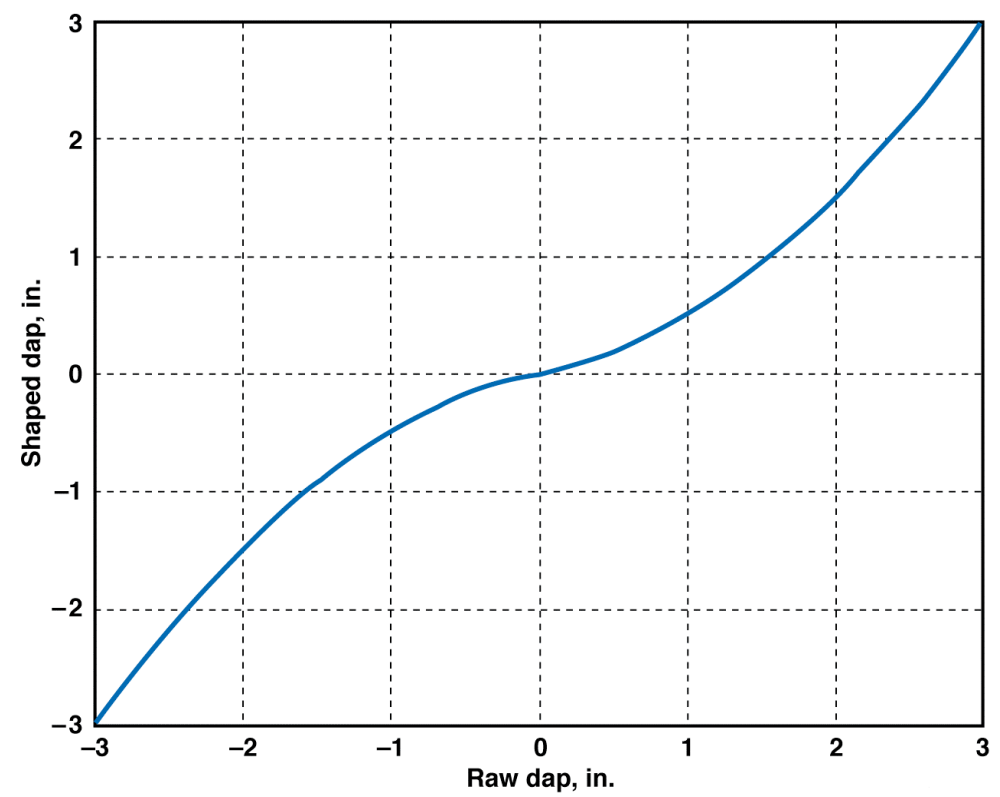

Figure 10. Illustration of lateral stick shaping.

No dead bands were implemented in the roll stick or the pitch stick. Their absence did not have any noticeable effect in the simulation; however, there were some noticeable effects seen in flight. This behavior is discussed in Ref. 13.

The final aspect of the roll axis design is gain scheduling $\mathrm{K}_{\mathrm{pe}}$ from Eq. (6). This gain was scheduled with angle of attack as seen in Fig. 11. The necessity to schedule this gain arises from the fact that as angle of attack increases the body axis yaw rate becomes a larger contributor to a stability axis roll. As a result, high-rate stability axis rolls 
require ample yaw control power. It turns out that this yaw control power becomes the limiting factor for stability axis rolls with moderate to high angles of attack. Reducing $\mathrm{K}_{\mathrm{pe}}$ as angle of attack increases helps constrain the stability axis roll command such that the vehicle has adequate yaw control power to perform the desired maneuver. This change in roll axis gain does not adversely affect the predictability of the control from a handling qualities standpoint.

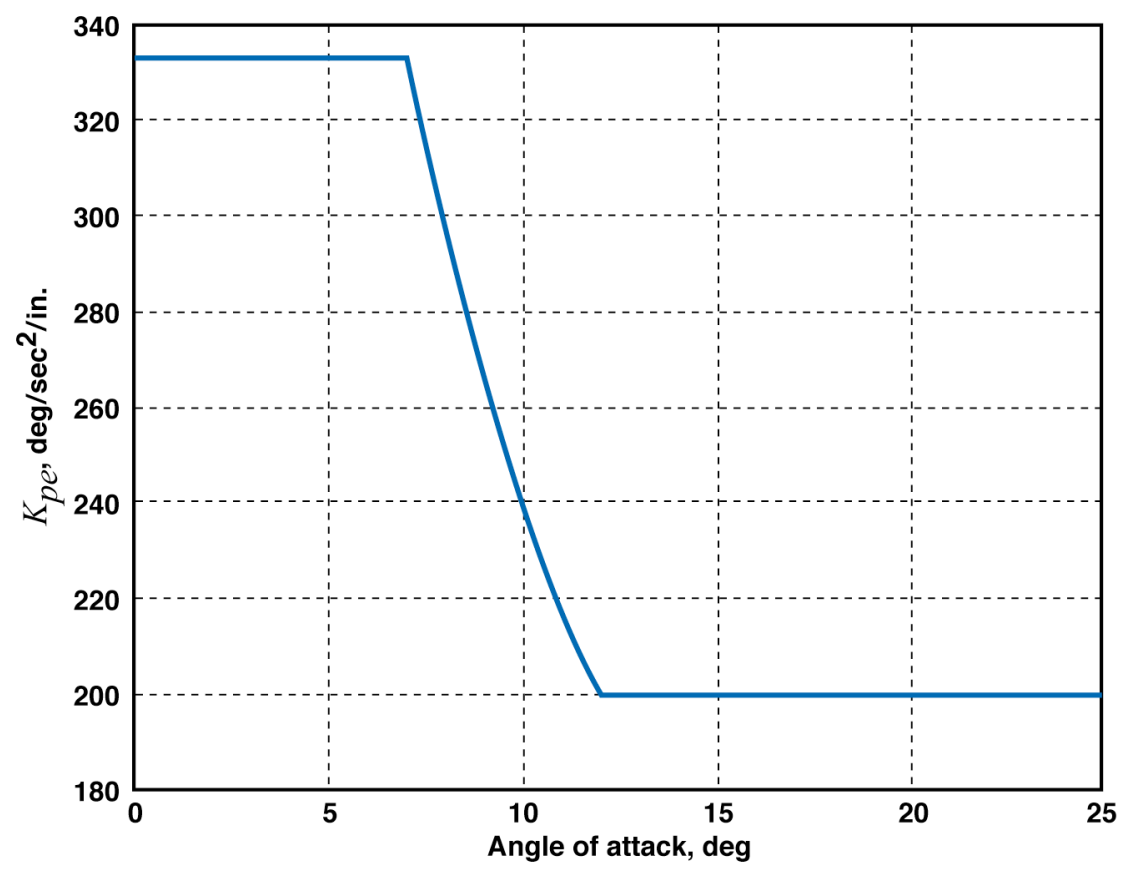

Figure 11. Illustration of lateral gain scheduling.

The yaw reference model is used to coordinate rolls $(\dot{\beta} \approx 0)$, and to initiate yawing motion to generate the lateral acceleration commanded by the pilot through the rudder pedals. A first order filter, Eq. (7), has been implemented to smooth this stability axis yaw rate command and differentiate it for use in the dynamic inversion.

$$
\frac{\dot{r}_{s, r e f}}{r_{s, c}}=\frac{\omega_{r^{s}}}{s+\omega_{r}}
$$

Equations (8)-(11) show how the desired $\dot{r}_{S, c}$ is derived and computed:

$$
\begin{array}{r}
\dot{\beta}=-r_{S}+\frac{1}{m V}\left[D \sin \beta+Y \cos \beta-X_{T} \cos \alpha \sin \beta+Y_{T} \cos \beta-Z_{T} \sin \alpha \sin \beta\right] \\
+\frac{g}{V}[\cos \alpha \sin \beta \sin \theta+\cos \beta \sin \varphi \cos \theta-\sin \alpha \sin \beta \cos \varphi \cos \theta]
\end{array}
$$

Assume $\beta \approx 0$, and thrust effects are small:

$$
\dot{\beta} \approx-r_{S}+\frac{Y}{m V}+\frac{g}{V} \sin \varphi \cos \theta=-r_{S}+\frac{g}{V}\left(a_{n y}+\sin \varphi \cos \theta\right)
$$

Set $(\dot{\beta} \approx 0)$ and solve for yaw rate command: 


$$
\begin{gathered}
r_{s, c}=\frac{g}{V}\left(a_{n y, c}+\sin \varphi \cos \theta\right) \\
r_{s, c}=\frac{g}{V}\left(k_{d r p} d r p+\sin \varphi \cos \theta\right)
\end{gathered}
$$

Getting good tracking in the yaw axis without feeding back sideslip angle can be difficult, but since sideslip is a difficult quantity to measure accurately it is often necessary to implement control laws that do not require feedback of this fundamental quantity. Replacing the commanded lateral acceleration with a proportional error feedback component, Eq. (12), has been shown via both simulation testing and flight to improve coordination for roll maneuvers. This additional gain and the feedback of measured lateral acceleration help to drive the measured lateral acceleration to the commanded value, improving the yaw characteristics of the control law.

$$
r_{s, c}=\frac{g}{V}\left(k_{a n y}\left[k_{d r p} d r p-a_{n y}\right]+\sin \varphi \cos \theta\right)
$$

One additional important characteristic of a lateral acceleration command architecture is that as dynamic pressure decreases, more sideslip is required to achieve the same lateral load factor. To account for this behavior, the rudder pedal gain, $\mathrm{k}_{\mathrm{drp}}$, has been scheduled with dynamic pressure as illustrated in Fig. 12. This gain scheduling was tuned empirically to produce lateral acceleration commands that generated sideslip angles of less than $7^{\circ}$ for maximum pedal deflection throughout the envelope in Fig. 9.

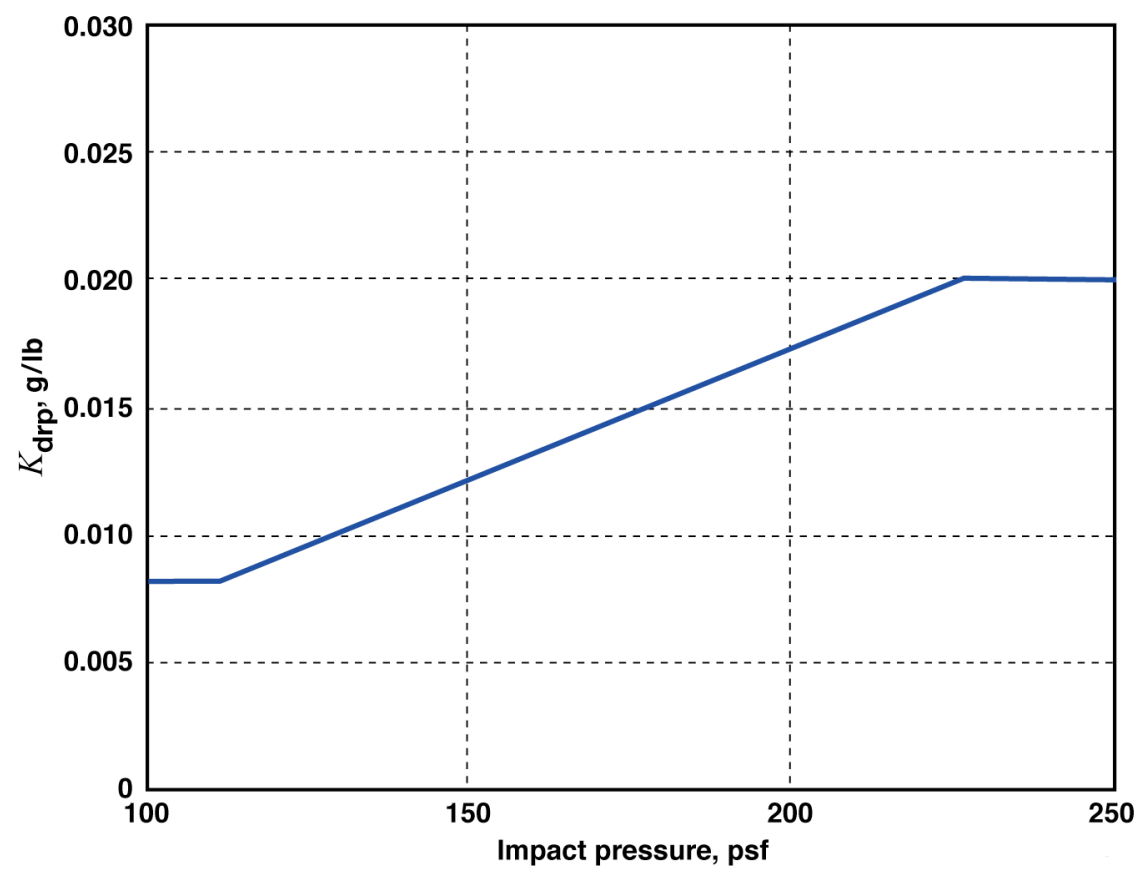

Figure 12. Illustration of rudder pedal gain scheduling.

An important characteristic of actuator dynamics as they relate to reference models and closed-loop tracking is that real actuators have the effect of increasing the natural frequency and reducing the damping of the closed-loop response when compared to the closed-loop responses with perfect actuators, as shown in Fig. 13. This behavior is a result of the actuator positions lagging behind the commands and causing overshoots of the angular rates commanded by the reference models. This side effect of the real actuators is easy to account for by detuning the natural frequencies of the command reference models. 


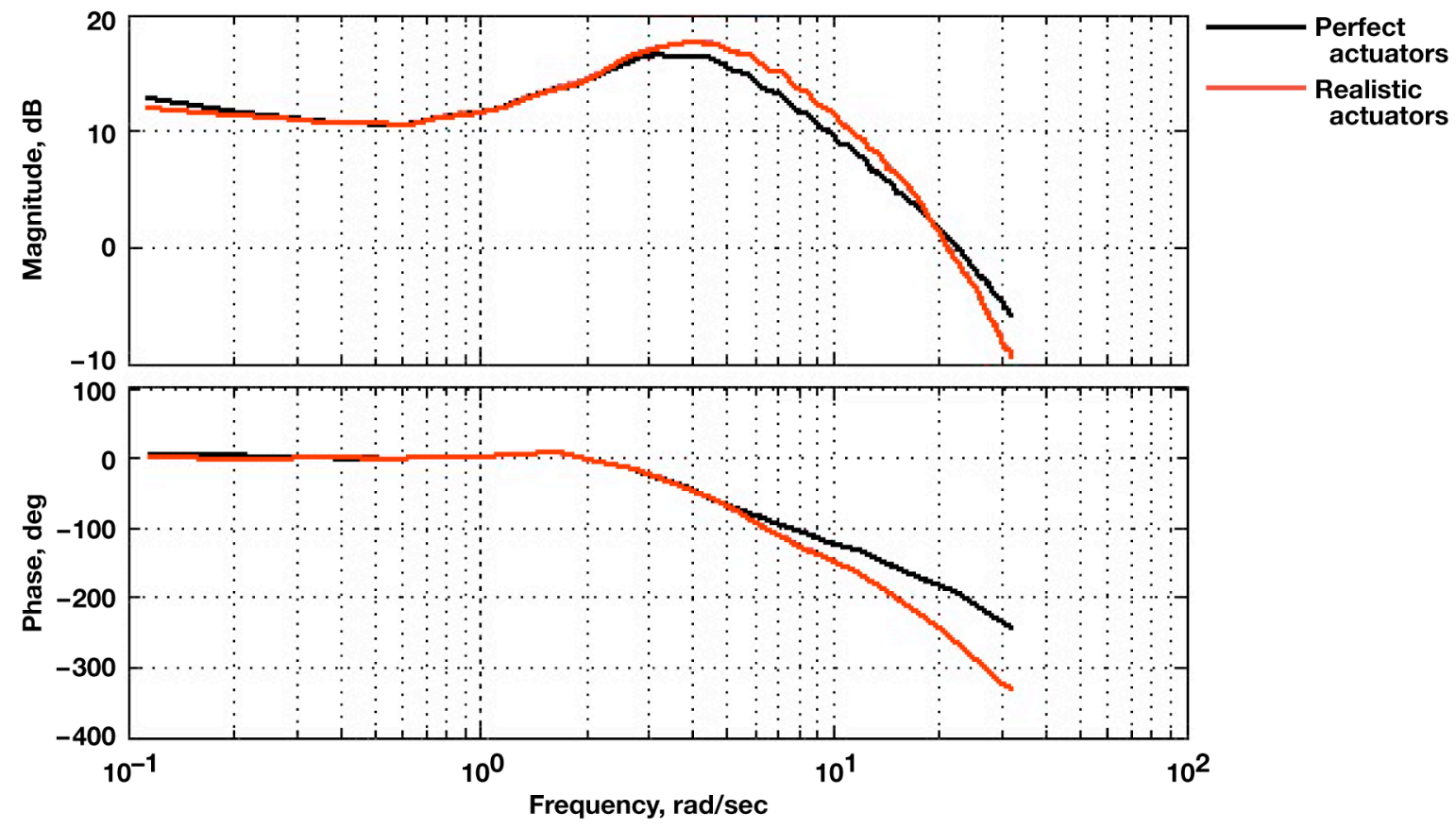

Figure 13. Illustration of the effect of actuator dynamics on closed-loop pitch tracking.

\section{Proportional-Plus-Integral Compensators}

The NDI control law must be robust enough to account for inaccuracies in the modeled plant dynamics and environmental disturbances that cannot be measured. A PI compensator has been implemented to provide the necessary robustness to these unmodeled or incorrectly modeled dynamics. The PI compensator has been tuned to have desirable disturbance rejection properties assuming that the dynamic inversion reduces the plant to a perfect integration. Figure 14 and Eqs. (13) and (14) are used to tune the PI compensators. The integral and proportional gains in Eq. (14) were chosen such that the poles of (14) match the poles of the reference model for each axis, noting that for first order reference models $k_{i}$ needs to be zero. This reference model matching is required for the stability proofs of the MRAC design discussed in Ref. 3.

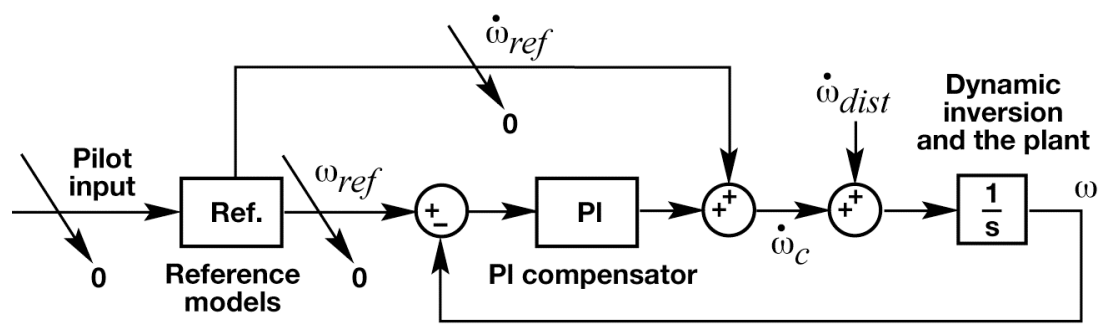

Figure 14. Block diagram for disturbance rejection.

$$
\left[-\left(k_{p}+\frac{k_{i}}{s}\right) \omega+\dot{\omega}_{\text {dist }}\right] \frac{1}{s}=\omega
$$




$$
\frac{\omega}{\dot{\omega}_{d i s t}}=\frac{1}{s+\left(k_{p}+\frac{k_{i}}{s}\right)}=\frac{s}{s^{2}+k_{p} s+k_{i}}
$$

\section{Structural Filters}

The signals that are filtered include: pitch rate, roll rate, yaw rate, lateral acceleration, and lateral stick. The filters implemented for this control law are identical to the baseline production structural filters. The primary effect of these filters from a control law standpoint for the frequencies of interest for rigid body dynamics is in the additional lag that they introduce into the feedback path. This lag is as much as 0.055 seconds in some loops. The specifics of these filters are not important for this discussion but their effect on the overall design is important. The additional lag that they introduce negatively impacts the phase margins discussed below. The lag also exacerbates the reduction in damping and increase in natural frequency of the closed-loop tracking discussed above in reference to actuators. These filters must be included when tuning the reference models.

\section{Simulation Predictions}

In addition to the actual flight asset, a simulation facility has been developed in support of F/A-18 airplane projects including FAST. The facility uses actual flight control computer hardware in addition to high-fidelity aerodynamics, actuator, and sensor models. This asset has been used extensively for both design and verification testing. The results presented below represent a mix of hardware-in-the-loop and all software simulations.

\section{A. Stability Margins and Robustness}

The application of linear stability margins to a nonlinear control law in a nonlinear flight environment does not provide any guarantees of stability or robustness. A common practice, ${ }^{20}$ however, is to evaluate fundamentally nonlinear problems in the linear regimen based on the assumption that the system behaves approximately linearly in a small region around equilibrium. Linear stability margins can be used to quantify the sensitivity of a nonlinear system to a class of linear uncertainties. Larger margins suggest that stability will likely be maintained in the presence of uncertainties that affect either the loop gain of the system or its phase. For example, large gain margins suggest that the system is robust in a stability sense to variations in surface effectiveness, and provide confidence that differences between flight and simulation control surface effectiveness values will not result in closed-loop instabilities.

A number of methods exist for computing the stability margins for a nonlinear system. The one chosen for this analysis is presented in Ref. 20. This method reconstructs the open-loop frequency response from closed-loop simulation or flight data by computing the frequency response of each element in a given loop and then recombining them to form the open-loop response. This method was chosen because it did not rely on linearizations of simplified models, and could be performed on flight data as well as simulation data.

The stability margins for the NDI control law have been evaluated throughout the envelope shown in Fig. 9 for a number of uncertain mass properties and with different aerodynamic models (table 1). While these test cases do not represent a significant class of robustness check cases, they are considered sufficient given the small flight envelope, well-known fidelity of the F/A-18 airplane simulation models, and the ability to quickly revert to the production control laws in flight should an instability arise.

Figure 15 shows the results for the pitch axis stability margins. Clearly, none of the stress cases at any of the flight conditions achieve the desired phase margin of $45^{\circ}$. This deficiency is not limiting in a practical sense because these margins are calculated using the high-fidelity nonlinear simulation that has been shown to correlate very well with flight results. Larger phase margins could be achieved with less aggressive pitch reference models, or by tuning the PI compensator. The MRAC, however, as discussed above, requires the PI compensator to be matched with the reference models, and detuning these reference models results in undesirable degradation of handling qualities. Modifying the structural filters, reducing sensor delays, and eliminating the ARTS command delay would also be means by which to recover phase margin; however, these are all outside the scope of this design. Otherwise, the margins appear to be well-behaved and do not vary significantly with flight condition, mass properties, or the aerodynamic model chosen. Additionally, there appears to be significant gain margin for all the test cases. This gain margin, combined with the flat nature of the phase response in the region surrounding the crossover frequency of the open-loop system (see Fig. 16), suggests that the system is robust to uncertainties in the pitch effectiveness of the control surfaces. 

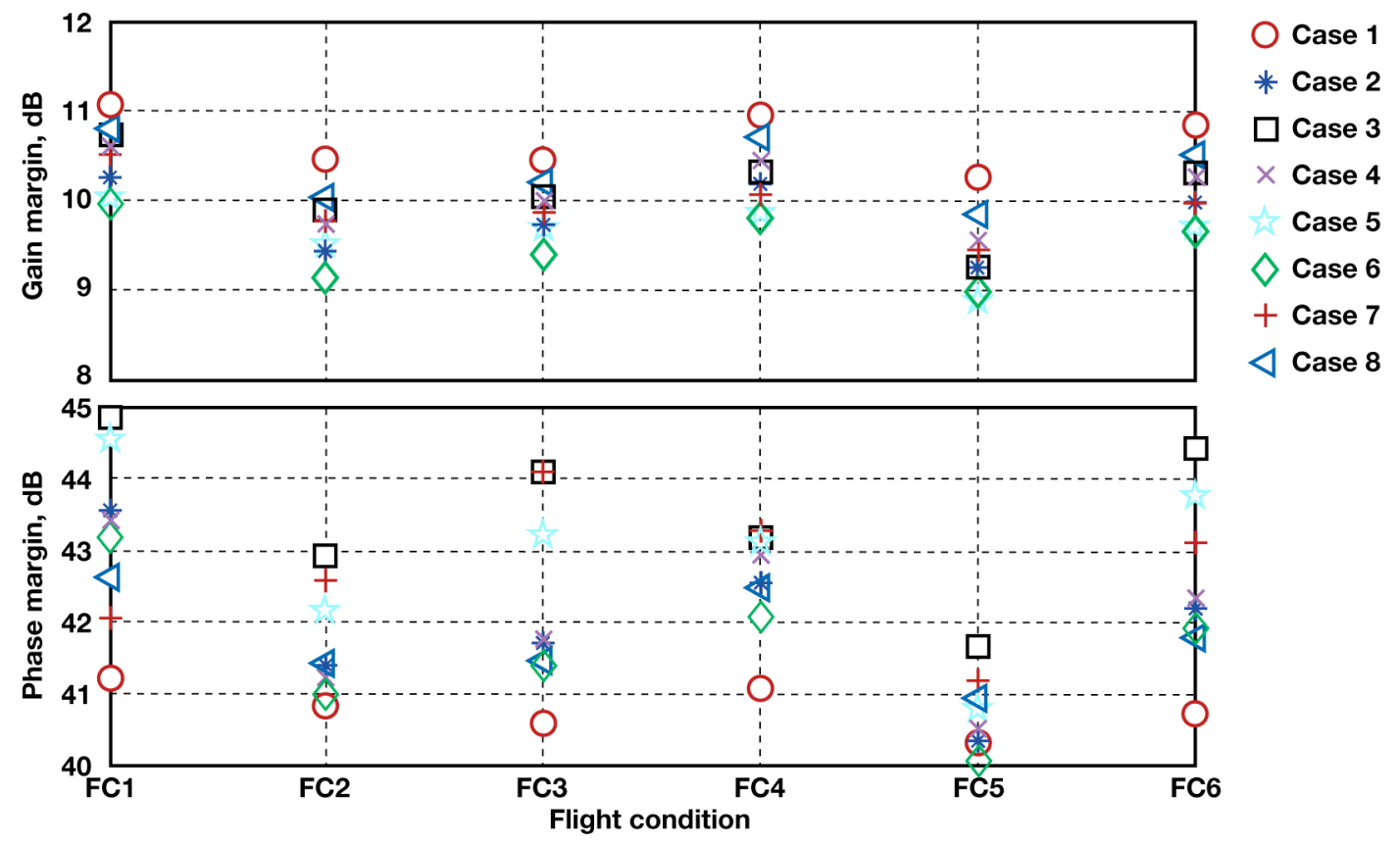

Figure 15. Pitch axis gain and phase margins.

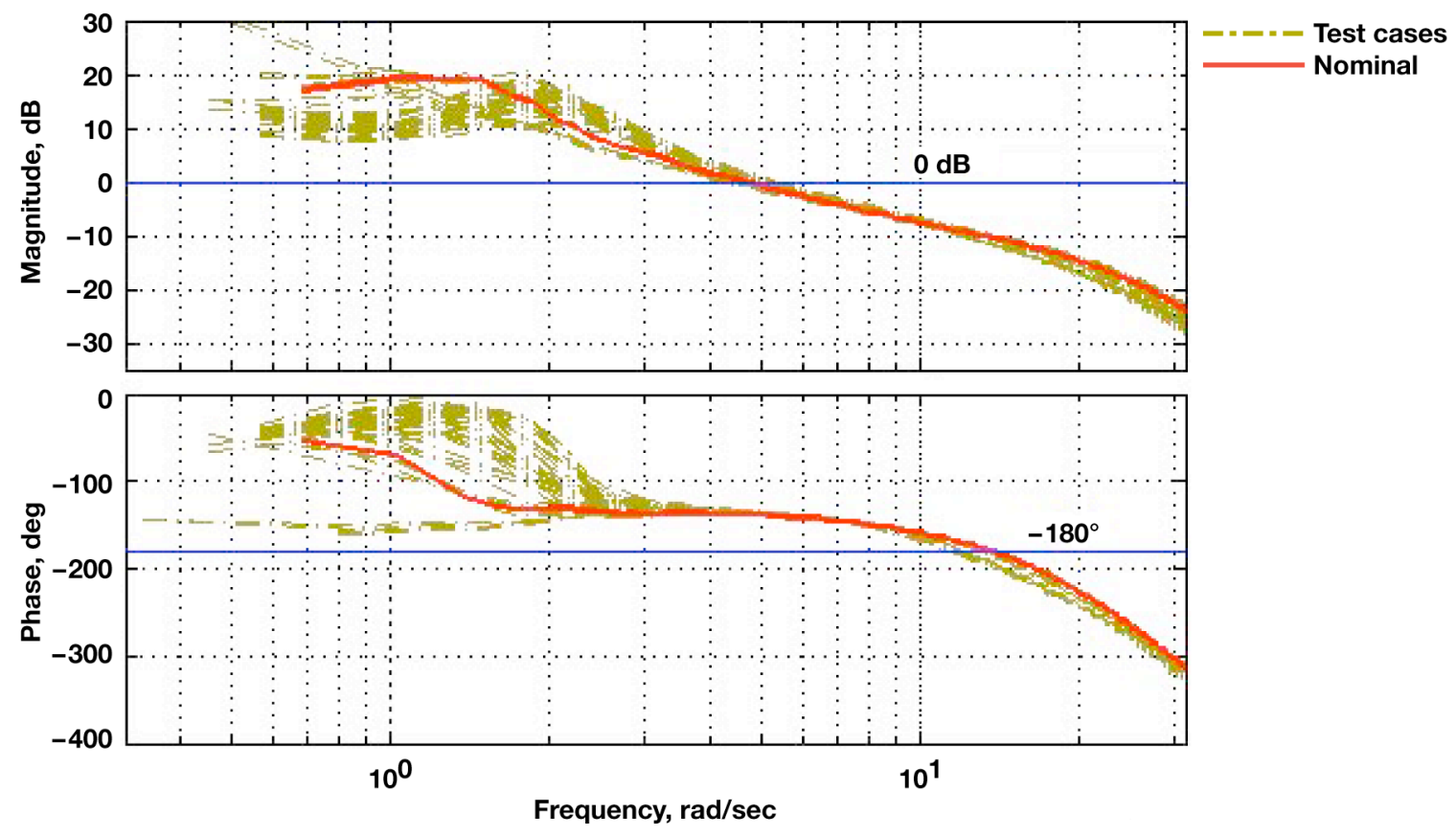

Figure 16. Pitch axis open-loop frequency responses.

Figure 17 presents the gain and phase margins in the roll loop. Both the phase and gain margins are well above the desired $45^{\circ}$ and $6 \mathrm{~dB}$. Similar to the pitch loop, the phase responses in roll (not shown) are flat in the region near the gain crossover, which suggests that the controller has good stability robustness in roll to inaccuracies in the roll surface effectiveness. The yaw axis margins look much the same as the roll axis margins, and are therefore not presented here. 


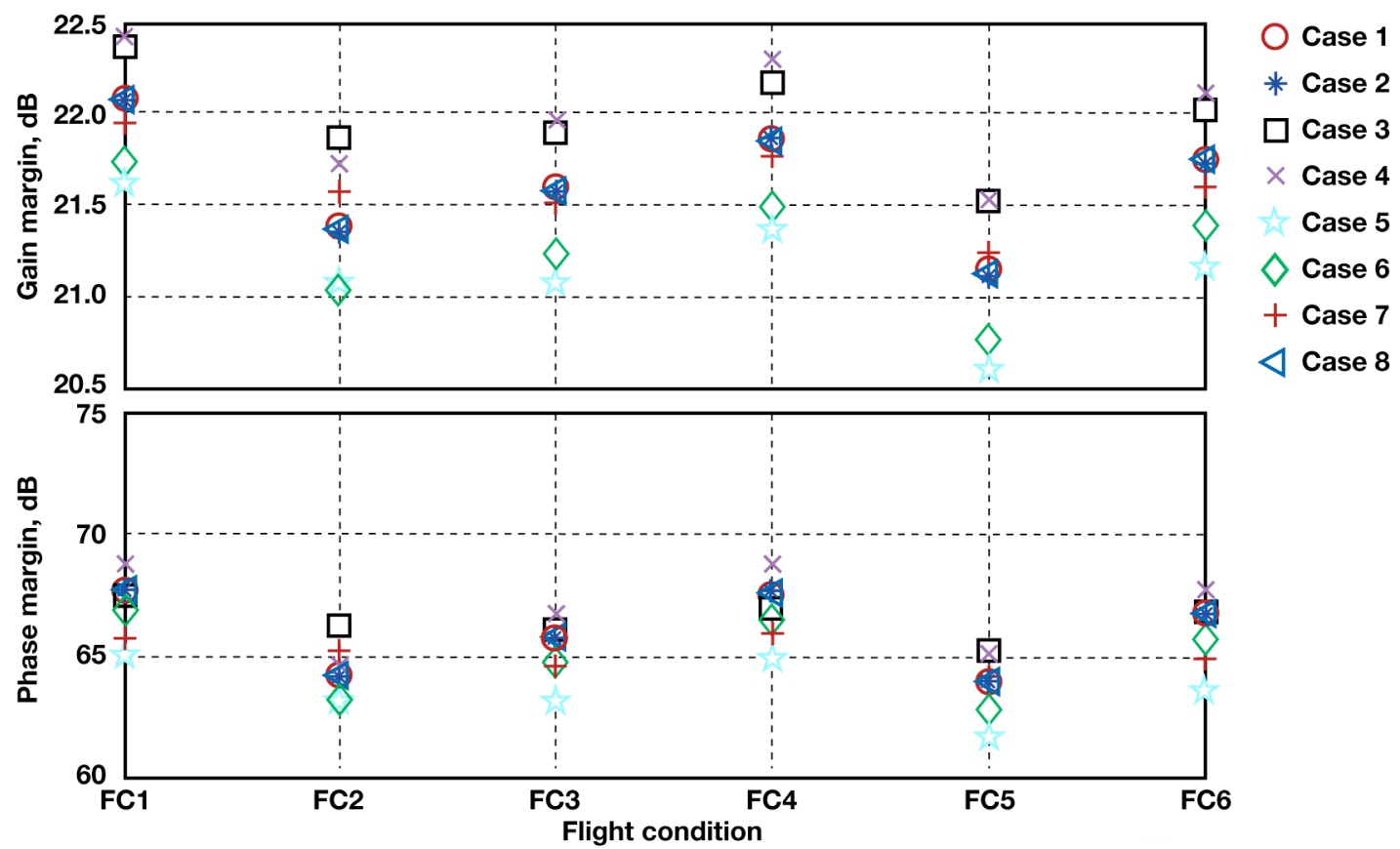

Figure 17. Roll axis gain and phase margins.

\section{B. Handling Qualities Predictions}

Many metrics exist for evaluating quantitatively the handling qualities of a given configuration. These metrics provide valuable insight into the behavior and predicted acceptability of a particular control architecture. They can also be used to highlight problem areas, such as PIO tendencies, prior to performing detailed and time-consuming pilot evaluations. Another advantage of using the metrics is that a large number of test cases can be run quickly to assess parametric sensitivities. The metrics that follow provide a solid basis for the belief that the control law presented herein will have desirable flying characteristics. This belief was confirmed both via piloted simulations and through flight test. ${ }^{13}$

All of the handling qualities metrics described below were used at each of the flight conditions shown in Fig. 9 and at each of the stress cases from table 1. A description of how handling qualities are evaluated can be found in Ref. 26.

\section{Reference Model Tracking}

The choice of the reference model forms was driven by the existence of a wealth of experience in the handling qualities of the standard reference model forms from Ref. 18. It follows from this basic assumption that good tracking of reference models with desirable handling qualities ${ }^{18,21,22,25}$ should result in the closed-loop system exhibiting satisfactory behavior.

Good tracking, from a handling qualities perspective, is defined such that the pilot either cannot distinguish between the actual system and the design reference model or such that he would give them equivalent ratings. ${ }^{23}$ Reference 23 establishes quantitative criteria for this property based on the pitch axis for a conventional aircraft with a center stick. It labels the region in which the handling qualities of a dynamic system will be unchanged from a pilot perspective as the maximum unnoticeable added dynamic (MUAD) envelopes.

Figure 18 shows the pitch axis results for all the test cases (table 1) at all the flight conditions for the NDI control law. At the higher frequencies, no real aircraft system is able to exhibit perfect second order responses. This breakdown of second order behavior can be seen in Fig. 18, and is a result of the limited bandwidth of real actuators causing both phase and magnitude roll-off. This behavior is expected and acceptable. It is clear that in general the

off-nominal test cases produce closed-loop frequency responses that do not lie within the MUAD envelopes. This could result in degraded handing qualities; however, this was not what was observed in the flight-test data. ${ }^{13}$ 


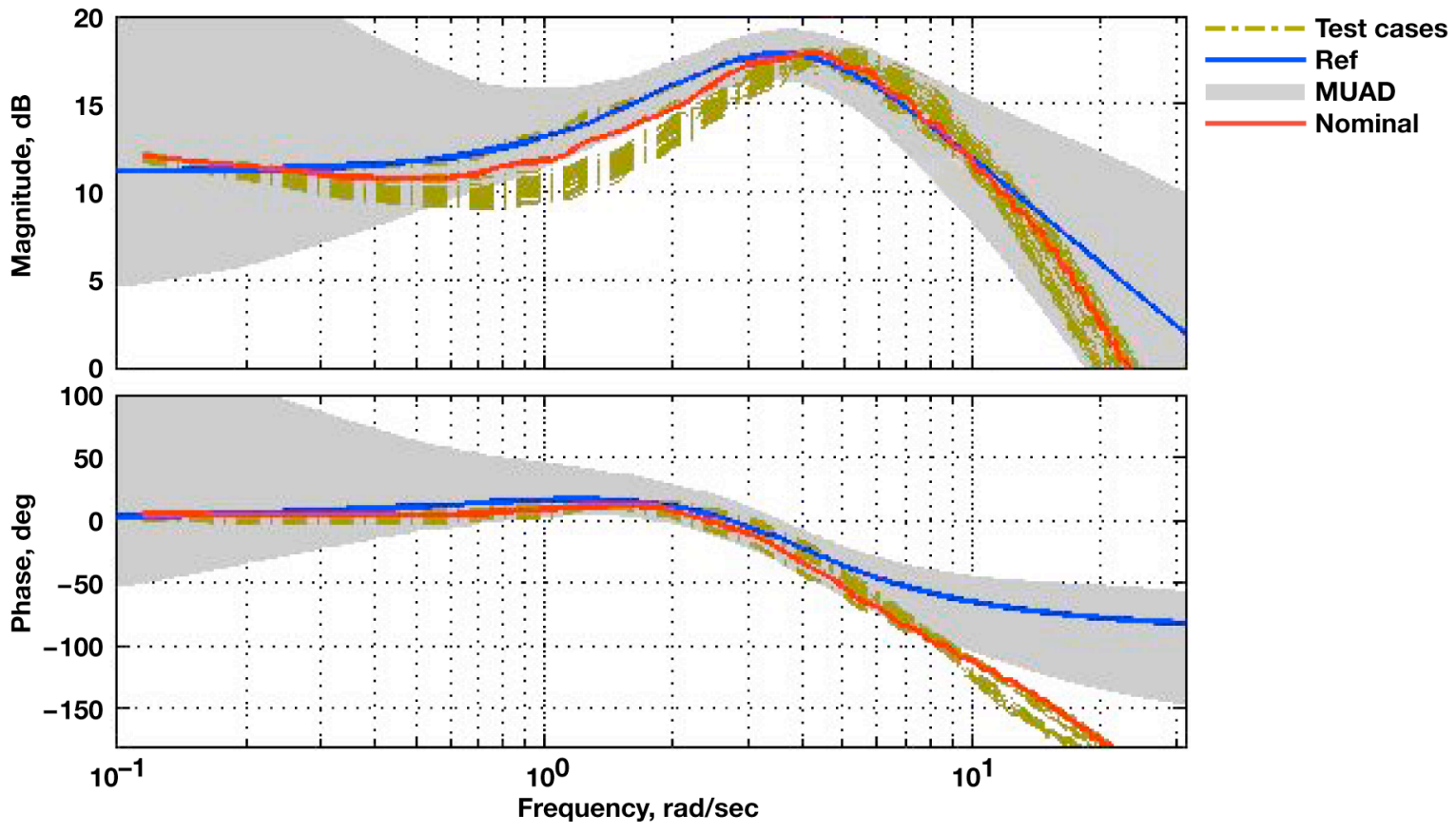

Figure 18. Pitch reference model tracking performance.

The roll axis was also examined to determine the closed-loop tracking performance. Figure 19 presents the results for the roll axis. The tracking in the roll axis is generally better than that of the pitch axis; however, it exhibits the same high frequency phase roll-off as the pitch axis, which was a result of actuator dynamics. Otherwise, the roll axis results suggest that good handling qualities will be observed in the roll axis. It should be noted that the MUAD envelopes do not strictly apply to the roll axis; however, they are included to give a point of reference with which to compare the tracking performance to the pitch axis.

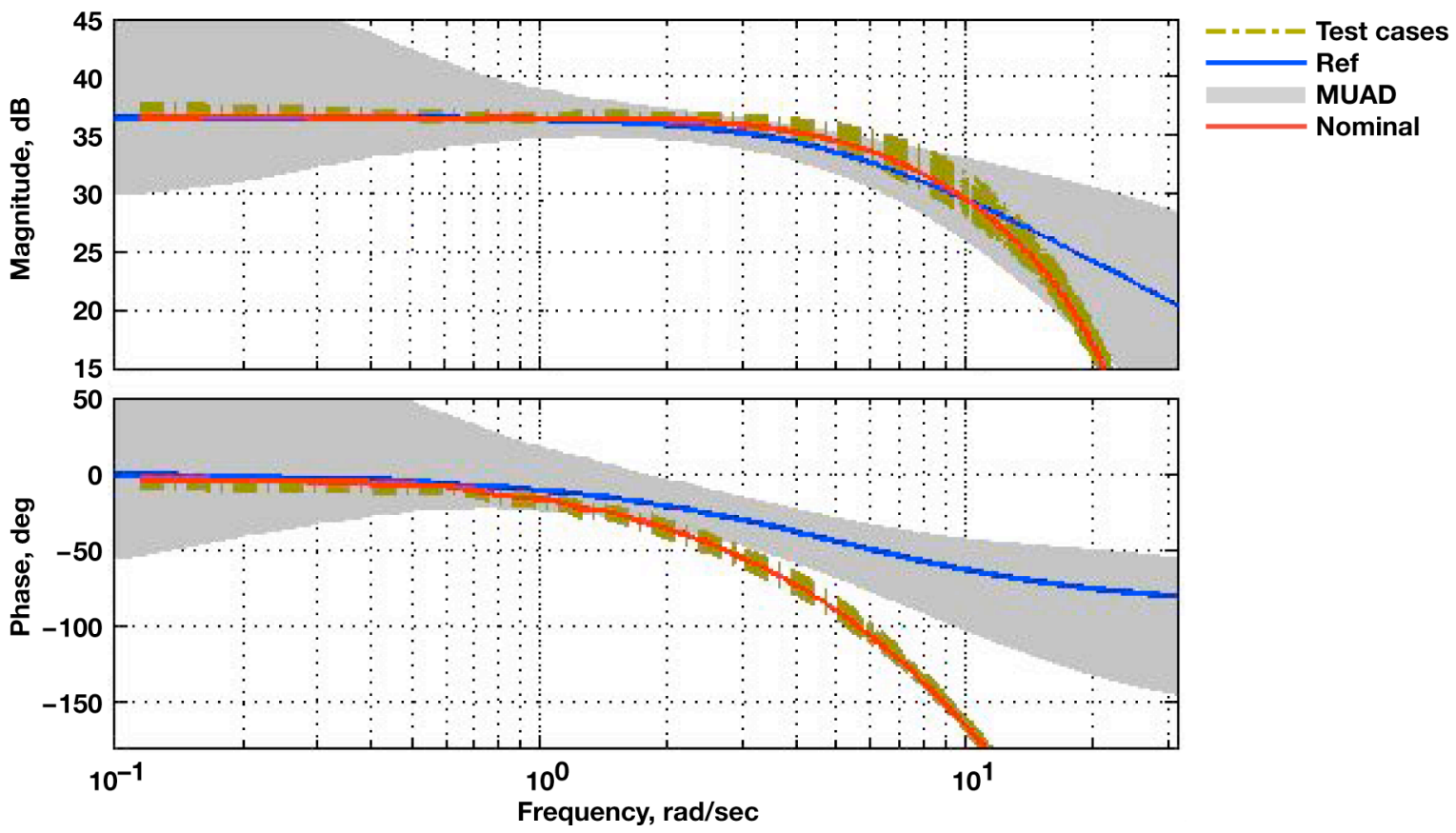

Figure 19. Roll reference model tracking performance.

American Institute of Aeronautics and Astronautics 
The yaw axis tracking is not presented herein because, as discussed previously, good tracking of the yaw reference model is not required for good yaw handling qualities. In general, the yaw tracking performance is poor due to the lack of angle of sideslip in the feedback and the aerodynamic calculations. Good roll maneuver coordination was observed despite poor yaw reference model tracking.

\section{Low Order Equivalent Systems (LOES)}

Low order equivalent systems (LOES) analysis approximates a parameterized model from test data. The parameterized model can then be used to assess the overall system from a handling qualities standpoint. ${ }^{24}$ This technique is the primary means for specifying longitudinal handling qualities requirements. ${ }^{18}$ The LOES technique is a natural way to evaluate the control law presented herein, as the dynamic inversion is designed in such a way as to track reference models of the exact form utilized by the LOES method.

The standard form for the short period from Ref. 18 is used for the parameterized model for the LOES analysis presented in Fig. 20. Additionally, the control anticipation parameter (CAP), as defined in Eq. (15), has also been shown to be useful in the evaluation of control systems for handling qualities. A detailed description of this approximation, the assumptions that underlie it, and its usage can be found in Refs. 18 and 19.
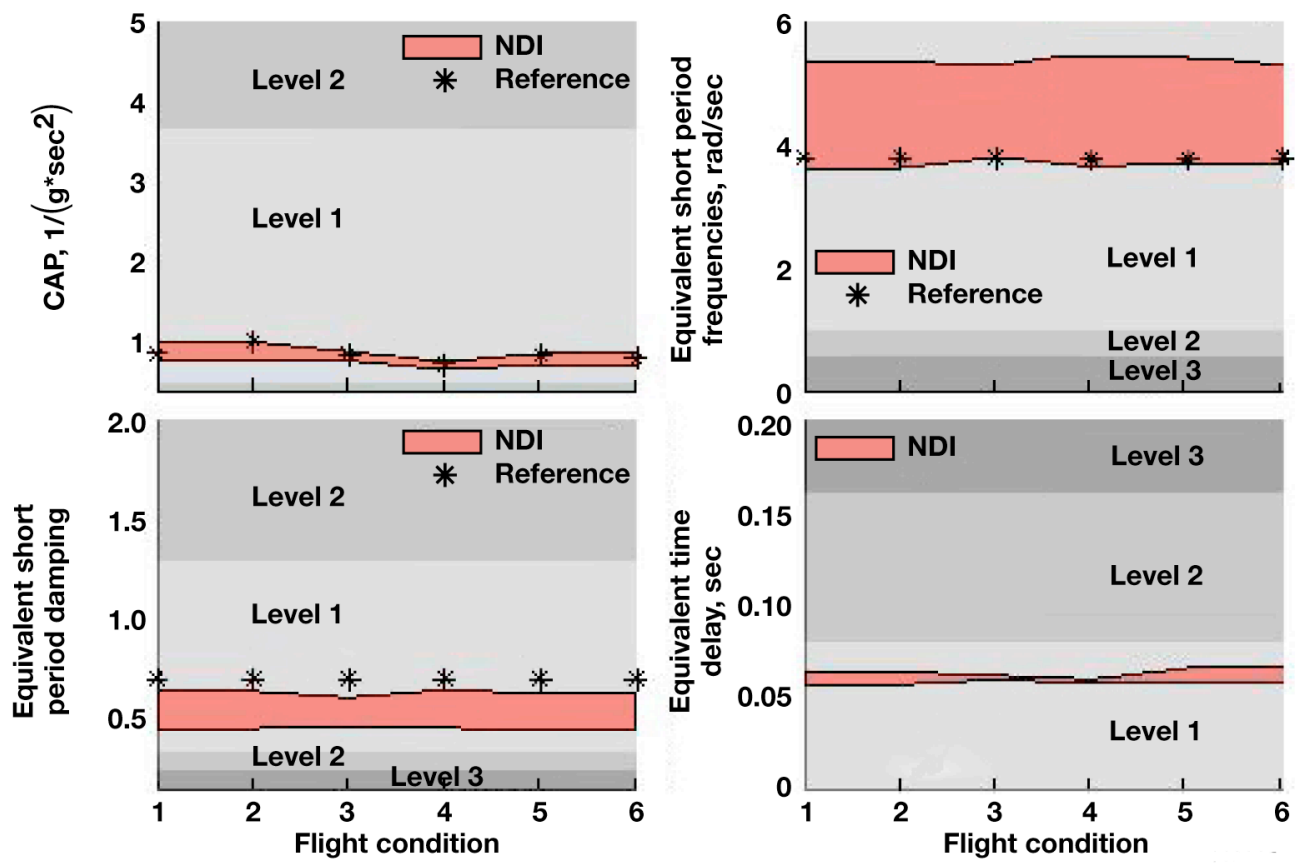

Figure 20. Pitch axis low order equivalent systems results.

$$
C A P=\frac{\omega_{s p}^{2} \frac{1}{L_{\alpha}} g}{V}
$$

Figure 20 presents the LOES results for the NDI handling qualities analyses graphically. The red envelopes represent the region within which the results for all of the test cases (table 1) for the NDI were contained, and the black asterisks represent the reference model design values.

Figure 20 shows the results for the control anticipation parameter, Eq. (15). It is apparent that the closed-loop response matches the desired reference model values very well, and that these values are by design in the heart of the level 1 region. This suggests that the NDI should exhibit excellent handling characteristics throughout the envelope shown in Fig. 9, even in the face of uncertainties in the aerodynamics and the mass properties (table 1).

Figure 20 also illustrates the results from a short period natural frequency standpoint. It is clear that the LOES-determined natural frequency is higher than the design natural frequency. This behavior is a result of the actuator dynamics, discussed in the control law description, and suggests that more detuning of the reference model 
natural frequency was needed to achieve the design response. Despite this undesirable difference, however, the closed-loop natural frequency lies in the heart of the level 1 region, and is therefore considered adequate.

The effect of actuator dynamics can also be seen on the short period damping ratio in Fig. 20. The actuator dynamics cause a slight reduction in the short period damping ratio when the LOES result is compared to the design reference model. From a LOES standpoint, this reduction in damping does not appear to affect the predicted handling qualities. This reduction in damping does, however, affect the magnitude of the resonant peak in Fig. 18; this will have detrimental effects that will be discussed below.

The final parameter of interest is the equivalent time delay. This parameter can best be understood as accounting for higher-order dynamics that are not modeled by the simple second order short period approximation. Lower values of this parameter are considered desirable because the pilot perceives this parameter as a pure delay. Large delays result in excessive pilot compensation and can cause PIO and other undesirable motion. It can be seen in Fig. 20 that the predicted time delay for the NDI is in the level 1 region. It is not shown here, but despite being in the level 1 region the NDI has a higher time delay than the baseline F/A-18 airplane control law. One of the drivers for this increase in equivalent time delay is the fact that the ARTS has an additional 0.0125 seconds of delay for longitudinal commands when compared to the baseline control law and control laws hosted in the RFCS. This extra delay is an artifact of the nature of the flight control system architecture and cannot be eliminated.

\section{Neal-Smith Analysis}

The Neal-Smith criterion is a mathematical-compensator-based tool for quantitatively assessing the predicted pilot workload required to accomplish a pitch tracking task. ${ }^{22}$ The mathematical compensator in Fig. 21 is tuned such that a particular closed-loop bandwidth is achieved while minimizing the resonant peak in the closed-loop response. The lead compensation from this compensator and the size of the resonant peak are then used to evaluate how difficult the task would likely be for a real pilot. Notable features of this analysis technique are that both the compensator lag ( 0.3 seconds in Fig. 21), and the desired bandwidth are parameters that can be varied. Generally, 0.3 seconds and $3.5 \mathrm{rad} / \mathrm{sec}$ are used for the lag and desired bandwidth, respectively, but variations in these parameters (particularly the bandwidth) can provide valuable insight into the sensitivity of the control law to aggressive piloting techniques. It is also desirable in some cases to use lag other than 0.3 seconds, which was in fact the case for the NDI.

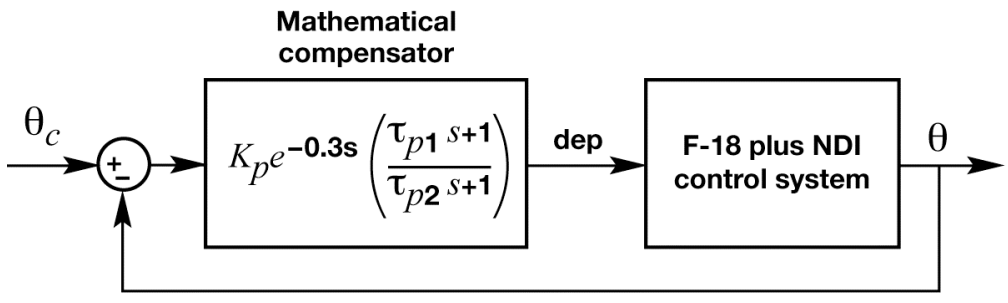

Figure 21. Neal-Smith compensator for the pitch tracking task.

Figure 22 shows how the NDI performs when evaluated with the Neal-Smith analysis method. The results in this figure were generated with deviations from the standard Neal-Smith criterion. The first and most obvious is that, as previously discussed, a range of bandwidths was evaluated. Each of the traces in Fig. 22 contains nine different bandwidths. The point at the far left side is $1.5 \mathrm{rad} / \mathrm{sec}$ and each successive point increases by $0.5 \mathrm{rad} / \mathrm{sec}$ up to $5.5 \mathrm{rad} / \mathrm{sec}$. This range more than encompasses the range expected for pilots, but the slope of each trace provides some confidence that the controller is not on the edge of having bad handling qualities. Another important difference is that instead of using 0.3 seconds for the lag, 0.2 seconds was used. This decision was made based on the results of analyzing the baseline F/A-18 control law (not shown). When the baseline F/A-18 control law was evaluated with the standard Neal-Smith criterion, it was found that a lag of 0.2 seconds matched the known level 1 characteristics of the control law better than the recommended value of 0.3 seconds. Therefore, 0.2 seconds was used to evaluate the NDI control law for all of the Neal-Smith analyses. 


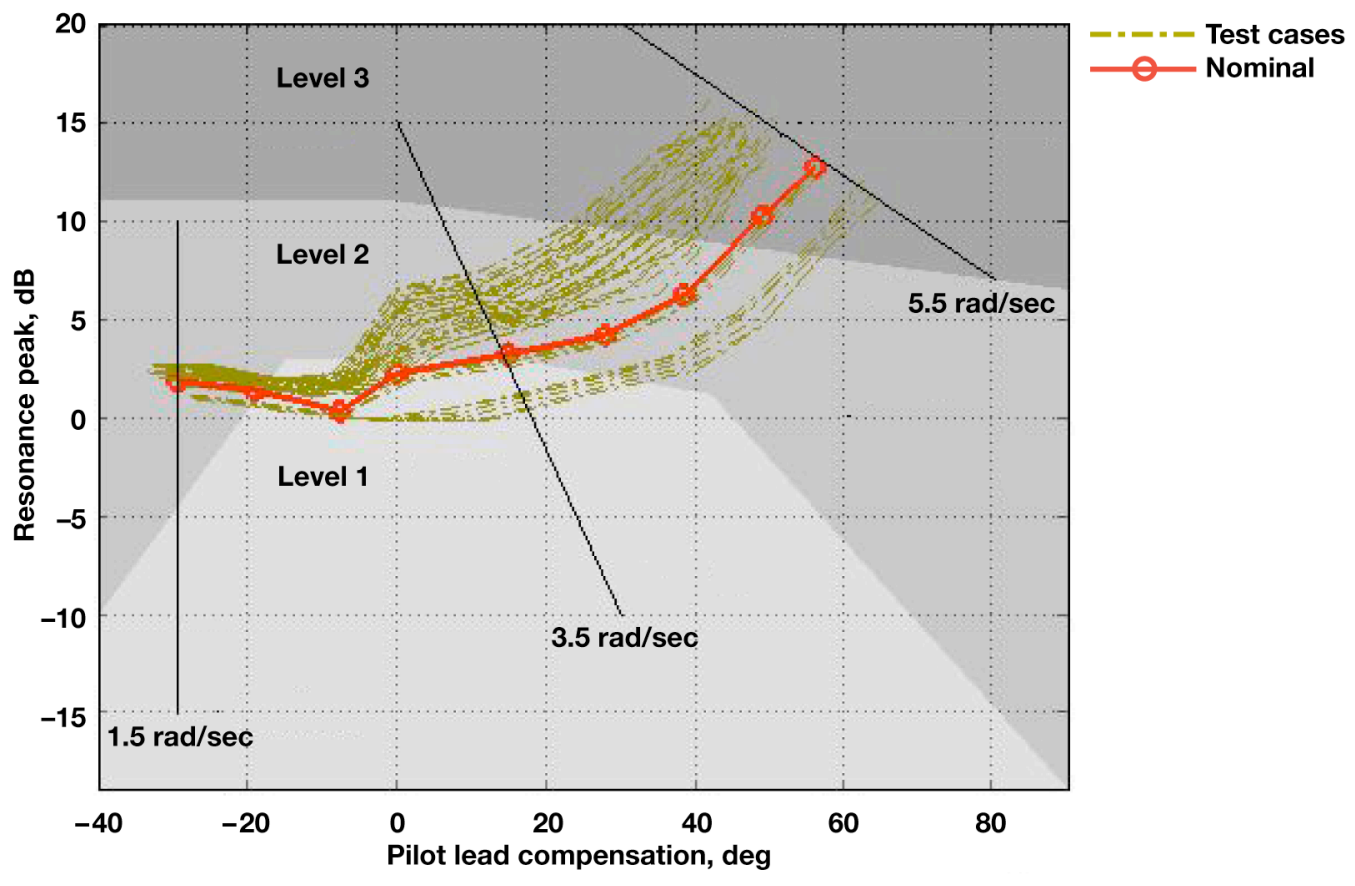

Figure 22. Neal-Smith criterion simulation predictions for nonlinear dynamic inversion.

Figure 22 shows that for the nominal configuration (flight condition 6, nominal center of gravity and inertias, and the design aerodynamic model) level 1 handling qualities are predicted for the range of bandwidths from $2 \mathrm{rad} / \mathrm{sec}$ to $3.5 \mathrm{rad} / \mathrm{sec}$. This result is exactly as desired. It is clear however, that deviations from the nominal configuration do produce some undesirable changes in handling characteristics. This deviation is expected, and the flight-test data will be used to determine if any changes to the control law are necessary to regain level 1 handling qualities. ${ }^{13}$

\section{Bandwidth Criterion Pitch}

Many handling qualities metrics depend on the bandwidth of the total system. ${ }^{25}$ Reference 25 outlines a metric that is based on a specific definition of the bandwidth determined from the pitch frequency response of the vehicle and control system. One major advantage of this metric is that it also includes a prediction of PIO. ${ }^{21}$ Neither Ref. 21 nor Ref. 25 contain the most up-to-date handling qualities level boundaries when the feel system is included; the boundaries from Ref. 18 were used for the analysis presented below.

This metric defines the bandwidth as the lesser frequency at which the phase angle passes through $-135^{\circ}\left(45^{\circ}\right.$ of phase margin) and the frequency at which there is $6 \mathrm{~dB}$ of gain margin. Also of importance is the definition of phase delay: the angle between $-180^{\circ}$ phase and the actual phase of the response at twice the frequency of the $-180^{\circ}$ phase crossover divided by 57.3 times that same frequency. See either Ref. 21 or Ref. 25 for a more complete description of these important parameters. One last important parameter for the analysis presented herein is the prominence of the resonant peak of the pitch rate frequency response (Fig. 18). If the prominence of the peak is greater than $9 \mathrm{~dB}$ a pitch bobble can be observed in even the level 1 region of Fig. 23. 


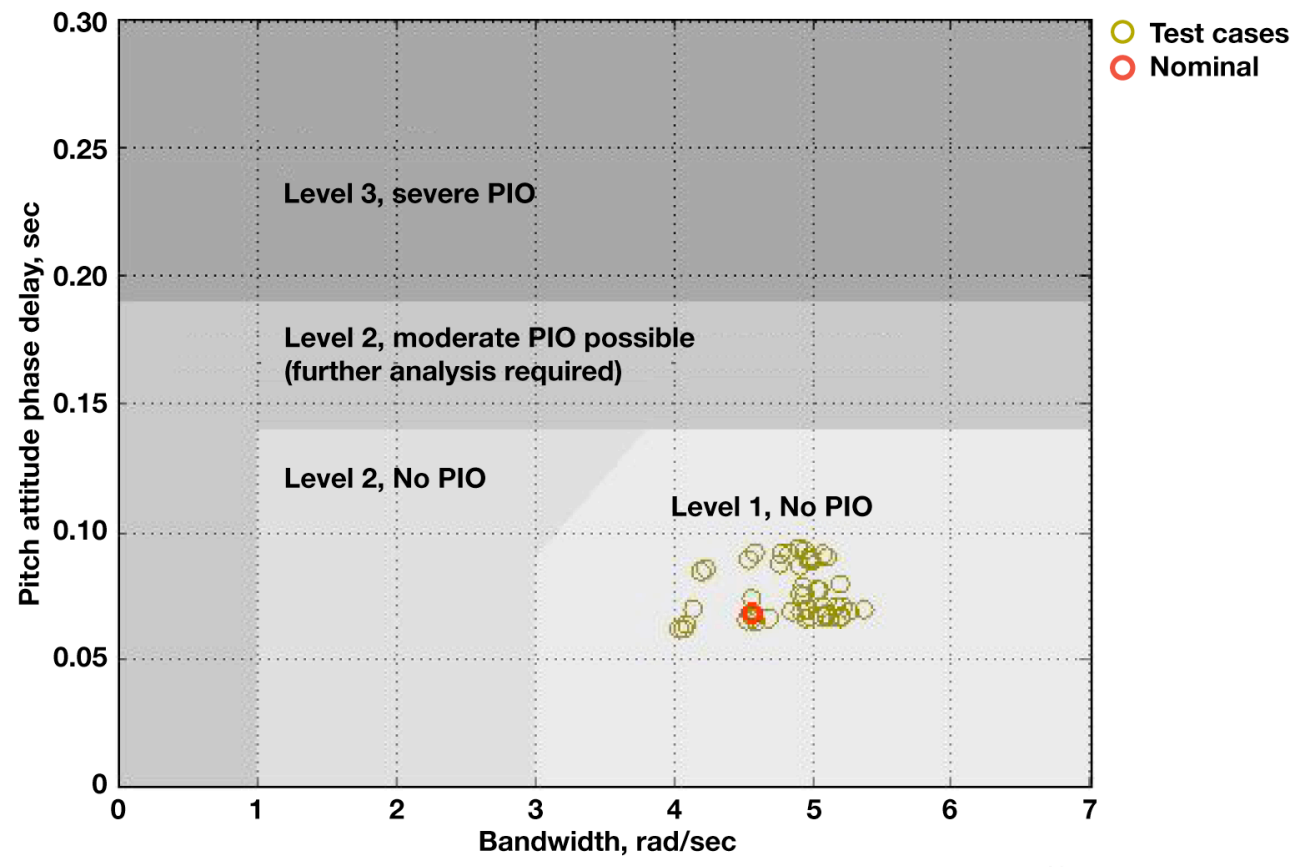

Figure 23. Simulation data evaluated against bandwidth criterion.

As can be seen in Fig. 23, the NDI is predicted to have excellent handling qualities regardless of the flight condition or case chosen from table 1. One subtle issue that was missed during the design, however, is related to the prominence of the resonant peak in Fig. 18. A closer look at Fig. 18 shows that many of the cases exceed the 9-dB limit called for in the bandwidth metric, and it was only after pilot comments regarding a pitch bobble in flight that this portion of the metric was fully appreciated. Many of the cases tested, including the nominal case, exhibited a mild pitch bobble for pitch attitude captures in both the simulation and in flight, and it is believed that this is related to the resonant peak prominence of $9 \mathrm{~dB}$. This over-pronounced resonant peak is likely due to the damping ratio reduction caused by actuator dynamics, and therefore could be addressed by tuning the pitch reference model.

\section{Roll and Yaw Handling Qualities Predictions}

Metrics do exist for evaluation of the roll and yaw axes; however, they proved less useful and less developed than the pitch axis metrics. Therefore, the decision was made to use a more heuristic and empirical approach in these axes. Based on pilot comments and opinions it was decided that rolls would be about the stability axis and that for most maneuvers the airplane should be flyable with the pilot's feet on the floor. As a result the primary function of the yaw axis of the control law is to maintain coordination. The rudder pedal is used only to generate lateral acceleration. The time constant of the roll mode and steady-state gain in the roll axis were tuned empirically in the simulation to yield good harmony with the pitch axis and minimize undesirable roll and yaw motions for closedloop tracking tasks, such as air-to-air tracking. The rudder pedal loop gain was also tuned empirically to produce desirable responses and characteristics during steady heading sideslip maneuvers.

\section{Conclusions}

The nonlinear dynamic inversion architecture outlined in this paper has been shown to be a straightforward structure for developing a model-following control law. The architecture is robust to a limited class of unmodeled or incorrectly modeled uncertainties, and is predicted to have level 1 handing qualities. The design should provide a solid baseline control law upon which advanced control elements can be built and tested in a flight environment.

The conservation of angular momentum formulation was found to be adequate to achieve level 1 predictions for handling qualities. Additionally, the aerodynamic formulation that ignores the moment contributions of drag, thrust, and structural flexibility was found to have no noticeable effect on the quality of the tracking, and the real-time computation of the simulation aerodynamic tables was found to be adequate even in the presence of some aerodynamic uncertainty. 
Utilizing the standard modal forms from MIL-STD-1797B to produce desired dynamics proved straightforward and effective. Implementing a pure angular rate feedback control structure presented challenges, but it was shown that adequate performance is attainable with proper tuning, shaping, and scheduling. The proposal was made that this control structure could be extended to larger envelopes with the proper scheduling of the transfer function parameters. Maintaining load factor during roll maneuvers for a pitch rate command system was found to be challenging, especially for inverted flight; however, the challenges could be addressed satisfactorily through kinematic manipulations and issuing commands about the stability axis. Turn coordination via cancellation of angle of sideslip derivative produced excellent results and was found not to require feedback of sideslip angle. Tuning the proportional integral controller compensator gains to match the poles of the transfer functions was found to be a straightforward and effective approach for their design.

Accounting for actuator dynamics was found to be the primary handling qualities challenge, but this challenge could be addressed through proper tuning of the reference models and proportional integral controller compensators. The primary effects of the actuators were found to be phase loss at high frequencies, and an apparent increase in the natural frequency with a corresponding decrease in the damping ratio of the closed-loop responses. This reduction in damping was noticeable in the reference model tracking, but was most obvious when the simulation data was evaluated using low order equivalent systems analysis. While those metrics did not predict any handling qualities implications due to this reduction in damping, they in fact caused a pitch bobble that was noted in flight and in the piloted simulations, and which was accurately predicted by the bandwidth metric. This bobble was generally a mildly unpleasant deficiency from a pilot's standpoint. The Neal-Smith criteria predict good handling qualities throughout the envelope with no cliffs related to changes in control bandwidth.

The stability margins for the control law were found to be well-behaved and not extremely sensitive to small changes in aerodynamics or mass properties; however, the pitch-loop phase margin was found to be less than $45^{\circ}$, which was an undesirable result. Many factors contributed to this low phase margin: structural filters, sensor dynamics, actuator dynamics, and the control hardware architecture. The high fidelity of the models used for the margin analysis coupled with the safe nature of the flight envelope provide the confidence that it is safe to proceed to flight with phase margins in the pitch axis between $40^{\circ}$ and $45^{\circ}$.

The weighted pseudo inverse was found to be a powerful tool for distributing control power to meet control objectives while accounting for outside constraints such as actuator dynamics and could be expanded to include constraints such as load limiting. The null space of the control effectiveness matrix (B) was found to yield an elegant and flexible set of trim surface combinations; external design goals could be used to decide between these trim solutions without producing any effects on transient responses.

A number of areas have been highlighted in which additional complexity could prove useful, including a reduction in the prominence of the resonant peak for the pitch axis reference model to reduce an undesirable pitch bobble, and addition of a steady-state pitch rate command to the pitch reference model such that one-g flight could be maintained for $360^{\circ}$ rolls. These minor design changes were not warranted for the current development, as the nonlinear dynamic inversion is predicted to have level 1 handing qualities without them.

\section{Appendix A: Detailed Equations for Dynamic Inversion}

$\begin{array}{ll}b & =\text { wing span } \\ C & =\text { coefficient } \\ d a & =\text { rolling aileron position } \\ d l e f & =\text { rolling leading edge flap position } \\ d r & =\text { yawing rudder position } \\ d s & =\text { rolling stabilator position } \\ d t e f & =\text { rolling trailing edge flap position } \\ s a & =\text { pitching aileron position } \\ \text { slef } & =\text { pitching leading edge flap position } \\ s r & =\text { pitching rudder position } \\ s s & =\text { pitching stabilator position } \\ \text { stef } & =\text { pitching trailing edge flap position } \\ x c m & =\text { longitudinal center of mass location (origin at the aerodynamic reference point) } \\ y c m & =\text { lateral center of mass location (origin at the aerodynamic reference point) } \\ z c m & =\text { vertical center of mass location (origin at the aerodynamic reference point) }\end{array}$




\section{Subscripts}

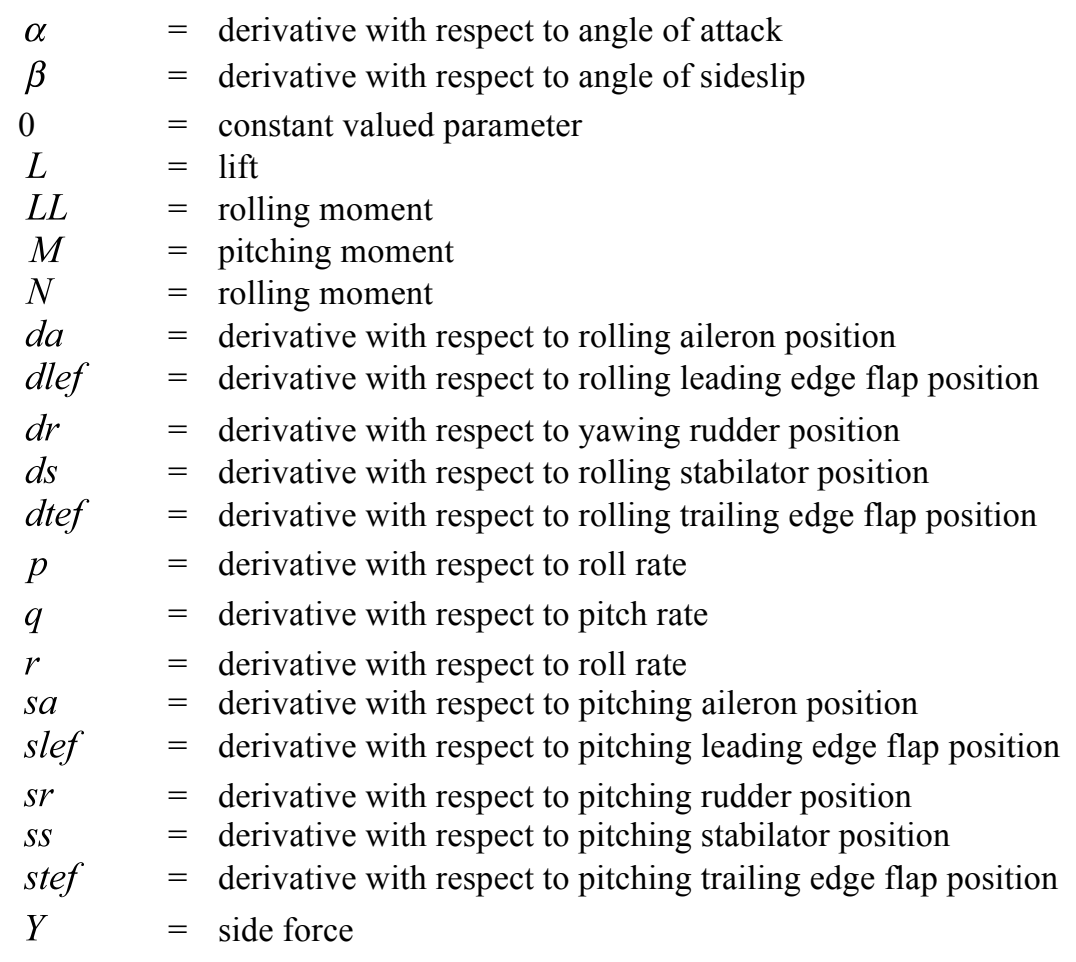

Starting with conservation of angular momentum in Eq. (16), the dynamic inversion can be derived from first principals with some important assumptions about the aerodynamic behavior, Eq. (17).

$$
\begin{gathered}
\vec{H}=I \overrightarrow{\omega^{b}} \\
\overrightarrow{\dot{H}^{e}}=\overrightarrow{I \dot{\omega}^{b}}+\overrightarrow{\omega^{b}} \times \overrightarrow{I \omega^{b}}=\left[\begin{array}{c}
L \\
M \\
N
\end{array}\right]=q_{C} S A x+q_{C} S B u
\end{gathered}
$$

The aircraft nonlinear aerodynamic moments $\mathrm{L}, \mathrm{M}$, and $\mathrm{N}$ from the above equation can be modeled using a linear combination of the contributions from each state variable and each input. For example, the rolling moment can be modeled as follows in Eqs. (18)-(21):

$$
L=q_{C} S\left[b C_{L L}+z_{c m} C_{Y}+y_{c m} C_{L}\right]
$$

Where,

$$
\begin{aligned}
& C_{L L}=C_{L L 0}+C_{L L \alpha} \alpha+C_{L L \beta} \beta+C_{L L p} p+C_{L L q} q+C_{L L r} r+C_{L L s a^{s a}}+C_{L L s s} s s+C_{L L s r} s r+ \\
& C_{L L s l e f} s l e f+C_{L L s}+C_{L L d a} d a+C_{L L d s} d s+C_{L L d r} d r+C_{L L d l e f} d l e f+C_{L L d t e f} d t e f
\end{aligned}
$$




$$
\begin{aligned}
& C_{Y}=C_{Y 0}+C_{Y \alpha} \alpha+C_{Y \beta} \beta+C_{Y p} p+C_{Y q} q+C_{Y r} r+C_{Y s a} s a+C_{Y s s} s s+C_{Y s r} s r+C_{Y s l e f} \text { slef }+ \\
& C_{Y s t e f} s^{s t e f}+C_{Y d a} d a+C_{Y d s} d s+C_{Y d r} d r+C_{Y d l e f} d l e f+C_{Y d t e f} d t e f \\
& C_{L}=C_{L 0}+C_{L \alpha} \alpha+C_{L \beta} \beta+C_{L p} p+C_{L q} q+C_{L r} r+C_{L s a} s a+C_{L s s} s s+C_{L s r} s r+C_{L s l e f} s l e f+ \\
& C_{L s t e f} \text { stef }+C_{L d a} d a+C_{L d s} d s+C_{L d r} d r+C_{L d l e f} d l e f+C_{L d t e f} d t e f
\end{aligned}
$$

All of these coefficients, which for this implementation were taken from table lookups of the aerodynamic database for the F/A-18 nonlinear simulation, can be combined into matrices and used to formulate the aircraft rotational dynamics as follows in Eqs. (22)-(29) and can be used to calculate the necessary control commands through the use of a weighted pseudo inverse:

$$
\begin{gathered}
\overrightarrow{\dot{\omega}^{b}}+\overrightarrow{\omega^{b}} \times I \overrightarrow{\omega^{b}}=q_{c} S A x+q_{c} S B u \\
q_{c} S B u=I \overrightarrow{\dot{\omega}_{c}^{b}}+\overrightarrow{\omega^{b}} \times I \overrightarrow{\omega^{b}}-q_{c} S A x \\
u=w_{p}^{-1} B^{T}\left(B w_{p}^{-1} B^{T}\right)^{-1}\left[\frac{1}{q_{c} S}\left(I \dot{\omega}_{c}^{b}+\overrightarrow{\omega^{b}} \times I \omega^{b}\right)-A x\right]
\end{gathered}
$$

Where,

$$
\begin{gathered}
x=\left[\begin{array}{l}
1 \\
\alpha \\
\beta \\
p \\
q \\
r
\end{array}\right], \text { and } u=\left[\begin{array}{l}
s a \\
s s \\
s r \\
s l e f \\
\text { stef } \\
d a \\
d s \\
d r \\
d l e f \\
d t e f
\end{array}\right], \overrightarrow{\omega^{b}}=\left[\begin{array}{l}
p \\
q \\
r
\end{array}\right], \text { and } \overrightarrow{\dot{\omega}_{c}^{b}}=\left[\begin{array}{l}
\dot{p}_{c} \\
\dot{q}_{c} \\
\dot{r}_{c}
\end{array}\right] \\
A=\left[\begin{array}{lllllll}
b 00 & { }_{c m} & y_{c m} \cos \alpha \\
0 c 0 & 0 & x_{c m} \cos \alpha \\
00 & b & -x_{c m} & 0
\end{array}\right]\left[\begin{array}{llllll}
C_{L L 0} & C_{L L \alpha} & C_{L L \beta} & C_{L L p} & C_{L L q} & C_{L L r} \\
C_{M 0} & C_{M \alpha} & C_{M \beta} & C_{M p} & C_{M q} & C_{M r} \\
C_{N 0} & C_{N \alpha} & C_{N \beta} & C_{N p} & C_{N q} & C_{N r} \\
C_{Y 0} & C_{Y \alpha} & C_{Y \beta} & C_{Y p} & C_{Y q} & C_{Y r} \\
C_{L 0} & C_{L \alpha} & C_{L \beta} & C_{L p} & C_{L q} & C_{L r} \\
& & & &
\end{array}\right]
\end{gathered}
$$




$$
\begin{gathered}
B=\left[\begin{array}{ccccc}
b & 0 & 0 & z_{c m} & y_{c m} \cos \alpha \\
0 & c & 0 & 0 & x_{c m} \\
0 & 0 & b & -x_{c m} & 0
\end{array}\right] * \\
{\left[\begin{array}{cccccccccc}
C_{L L s a} & C_{L L s s} & C_{L L s r} & C_{L L s l e f} & C_{\text {Lstef }} & C_{L L d a} & C_{L L d s} & C_{L L d r} & C_{\text {Ldlef }} & C_{\text {LLdtef }} \\
C_{M s a} & C_{M s s} & C_{M s r} & C_{M s l e f} & C_{M s t e f} & C_{M d a} & C_{M d s} & C_{M d r} & C_{M d l e f} & C_{M d t e f} \\
C_{N s a} & \vdots & \vdots & \vdots & \vdots & \vdots & \vdots & \vdots & \vdots & \vdots \\
C_{Y s a} & \vdots & \vdots & \vdots & \vdots & \vdots & \vdots & \vdots & \vdots & \vdots \\
C_{L s a} & \vdots & \vdots & \vdots & \vdots & \vdots & \vdots & \vdots & \vdots & C_{\text {Ldtef }}
\end{array}\right]}
\end{gathered}
$$

Assume symmetry about the $x z$ plane:

$$
\begin{aligned}
& I=\left[\begin{array}{ccc}
I_{x x} & 0 & -I_{x z} \\
0 & I_{y y} & 0 \\
-I_{x z} & 0 & I_{z z}
\end{array}\right] \\
& w_{p}=\operatorname{diag}\left[w_{s a} w_{s s} w_{s r} w_{s l e f} w_{s t e f}{ }^{w} d a^{w} d s^{w} d r{ }^{w} \text { dlef }{ }^{w} \text { dtef }\right]
\end{aligned}
$$




\section{References}

${ }^{1}$ Balas, G. J., "Flight Control Law Design: An Industry Perspective,” European Journal of Control, (2003)9, pp. 196-215.

${ }^{2}$ Bordignon, K., and Bessolo, J., "Control Allocation for the X-35B," AIAA-2002-6020, 2002.

${ }^{3}$ Hanson, C., Schaefer, J., Burken, J. J., Johnson, M. A., and Nguyen, N., "Handling Qualities Evaluations of Low Complexity Model Reference Adaptive Controllers for Reduced Pitch and Roll Damping Scenarios," AIAA-2011-\#\#\#\# (to be published), 2011.

${ }^{4}$ Schaefer, J., Hanson C., Johnson, M. A., and Nguyen, N., "Handling Qualities of Model Reference Adaptive Controllers with Varying Complexity for Pitch-Roll Coupled Failures," AIAA-2011-\#\#\#\# (to be published), 2011.

${ }^{5}$ Steinberg, M. L., “A Comparison of Intelligent, Adaptive, and Nonlinear Flight Control Laws,” AIAA-99-4044, 1999.

${ }^{6}$ Kaneshige, J., Bull, J., and Totah, J. J., "Generic Neural Flight Control and Autopilot System," AIAA-2000-4281, 2000.

${ }^{7}$ Rysdyk, R. T., and Calise, A. J., "Fault Tolerant Flight Control Via Adaptive Neural Network Augmentation," AIAA-98-4483, 1998

${ }^{8}$ Bosworth, J. T., "Flight Results of the NF-15B Intelligent Flight Control System (IFCS) Aircraft with Adaptation to a Longitudinally Destabilized Plant," AIAA-2008-6985, 2008.

${ }^{9}$ Bosworth, J. T., and Williams-Hayes, P. S., "Flight Results from the NF-15B Intelligent Flight Control System (IFCS) Aircraft with Adaptation to a Simulated Stabilator Failure," AIAA-2007-2818, 2007.

${ }^{10}$ Yedavalli, R. K., Shankar, P., and Doman, D. B., "Robustness Study of the Dynamic Inversion Based Indirect Adaptive Control of Flight Vehicles with Uncertain Model Data," Proceedings of the 2003 American Control Conference, 2003 , pp. 1005-1010.

${ }^{11}$ Frost, S. A., Taylor, B. R., Jutte, C. V., Burken, J. J., Trinh, K. V., and Bodson, M., “A Framework for Optimal Control Allocation with Structural Load Constraints," AIAA-2010-8112, 2010.

${ }^{12}$ Gregory, I. M., "Modified Dynamic Inversion to Control Large Flexible Aircraft - What's Going On?," AIAA-99-3998, 1999.

${ }^{13}$ Miller, C. J., "Nonlinear Dynamic Inversion Baseline Control Law: Flight Test Results," AIAA-2011-\#\#\#\# (to be published), 2011.

${ }^{14}$ Clarke, R., Allen, M. J., Dibley, R. P., Gera, J., and Hodgkinson, J., "Flight Test of the F/A-18 Active Aeroelastic Wing Airplane," AIAA-2005-6316, 2005.

${ }^{15}$ Enns, D. F., Bugajski, D. J., Pahle, J. W., Carter, J. F., Antoniewicz, R. F., Regenie, V., "Control Design and Flight Hardware Implementation Experience with Nonlinear, Dynamic Inversion Control (NASA-2) for the F/A-18 HARV," may be released to U.S. persons with NASA approval, High-Angle-of-Attack Technology Accomplishments, Lessons Learned, and Future Directions, NASA/CP-1998-207676/PT1.

${ }^{16}$ Georgie, J., and Valasek, J., "Evaluation of Longitudinal Desired Dynamics for Dynamic-Inversion Controlled Reentry Vehicles," Journal of Guidance, Control and Dynamics, Vol. 26, No. 5, September-October 2003, pp. 811-819.

${ }^{17}$ Cox, T. H, and Cotting, C. M., "A Generic Inner-Loop Control Law Structure for Six-Degree-of-Freedom Conceptual Aircraft Design,” NASA/TM-2005-212865, 2005.

${ }^{18}$ U.S. Department of Defense, Flying Qualities of Piloted Aircraft, MIL-STD-1797B, 15 February 2006.

${ }^{19}$ Roskam, J., Airplane Flight Dynamics and Automatic Flight Controls, Part II, Design, Analysis, and Research Corporation (DARcorporation), Lawrence, Kansas, 2003.

${ }^{20}$ Bosworth, J. T., and West, J. C., "Real-Time Open-Loop Frequency Response Analysis of Flight Test Data," AIAA-86-9738, 1986.

${ }^{21}$ Mitchell, D. G., and Field, E. J., "Nonlinearities and PIO with Advanced Aircraft Control Systems," RTO Meeting Proceedings 51 (RTO-MP-051), 2001, pp. 28-1-28-14.

${ }^{22}$ Neal, T. P., and Smith, R. E., "An In-Flight Investigation to Develop Control System Design Criteria for Fighter Airplanes," AFFDL-TR-70-74, Volume I, Air Force Flight Dynamics Laboratory, December 1970.

${ }^{23}$ Wood, J. R, and Hodgkinson, J., "Definition of Acceptable Levels of Mismatch for Equivalent Systems of Augmented CTOL Aircraft," MDC A6792, McDonnell Aircraft Company, St. Louis, Missouri, 1980.

${ }^{24}$ Hodgkinson, J., and LaManna, W. J., "Equivalent System Approaches to Handling Qualities Analysis and Design Problems of Augmented Aircraft," AIAA-1977-1122, 1977.

${ }^{25}$ Hoh, R. R., Mitchell, D. G., and Hodgkinson, J., "Bandwidth: A Criterion for Highly Augmented Airplanes," $A G A R D$ Criteria for Handling Qualities of Mil. Aircraft, 1982.

${ }^{26}$ Cooper, G. E., and Harper, Jr., R. P., The Use of Pilot Rating in the Evaluation of Aircraft Handling Qualities, NASA TN D-5153, 1969. 
Nonlinear Dynamic Inversion Baseline Control Law: Architecture and Performance Predictions

\author{
Chris Miller \\ Dryden Flight Research Center \\ Flight Control and Dynamics \\ 661-276-2902 \\ Chris.j.miller@nasa.gov
}




\section{Outline}

- Research Objectives

- Full-Scale Advanced Systems Testbed (FAST)

Background

- Control Law Description

- Theoretical Background and Implementation Details

- Reference Model Tracking

- Handling Qualities Predictions

- Conclusions

- Questions 


\section{Motivation for NDI}

Research Objectives

- RFI distributed in 2009 for research related to resilient aircraft control

- RFI responses discussed at AIAA GNC in 2009

- FAA, Academia, NASA, and Industry

- Three areas highlighted

- Investigate simple yet effective adaptive algorithms

- Explore pilot interactions with adaptive controllers

- Incorporate structural feedback into the flight control system
Why nonlinear dynamic inversion

- Utilized in literature for baseline control law for a number of adaptive and structural control techniques

- Explicit model following

- NDI contains explicit reference models, which integrate nicely with many adaptive control techniques, such as model reference adaptive control

- Failure modeling

- Fundamental-level failures (uncertainty in elements of the A- and B-matrices) can be modeled by altering the NDI reference models and PI compensators, and onboard aerodynamic tables

- Analysis formulation

- The NDI architecture is easy to analyze and include in stability proofs

- Open source architecture

- NDI is an open-source architecture. By specifying non-ITAR reference models, simulation and flight test data can be openly published 


\section{FAST Vehicle}

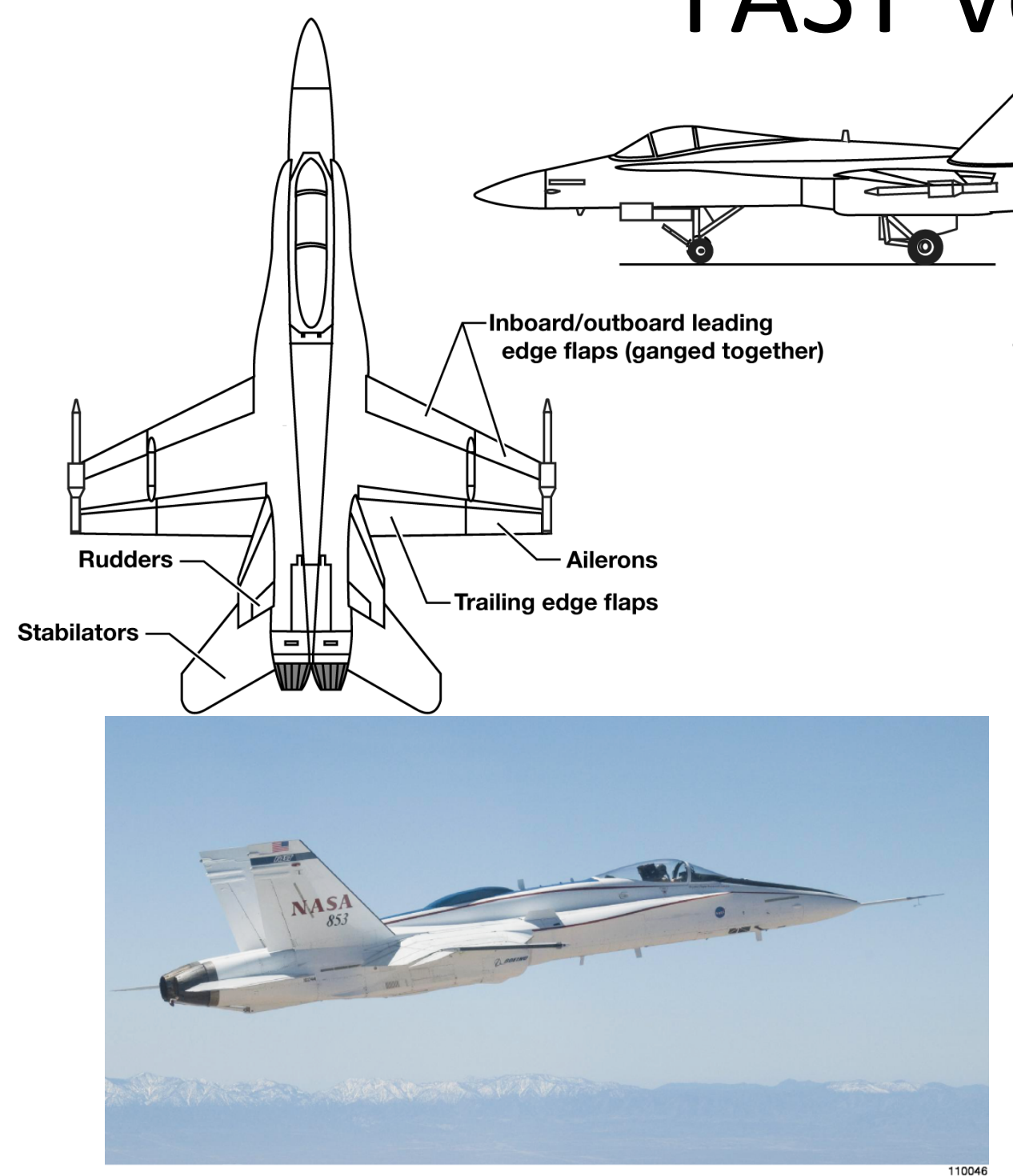

- Modified F/A-18

- Research flight control computers

- Quad RFCS

- Dual ARTS

$\overline{110047}_{0}$ Research instrumentation

- Research EGI

- Static and dynamic structural instrumentation

- FDMS

- Strain

- Accelerometers

- Nose boom 


\section{FAST Hardware Background}

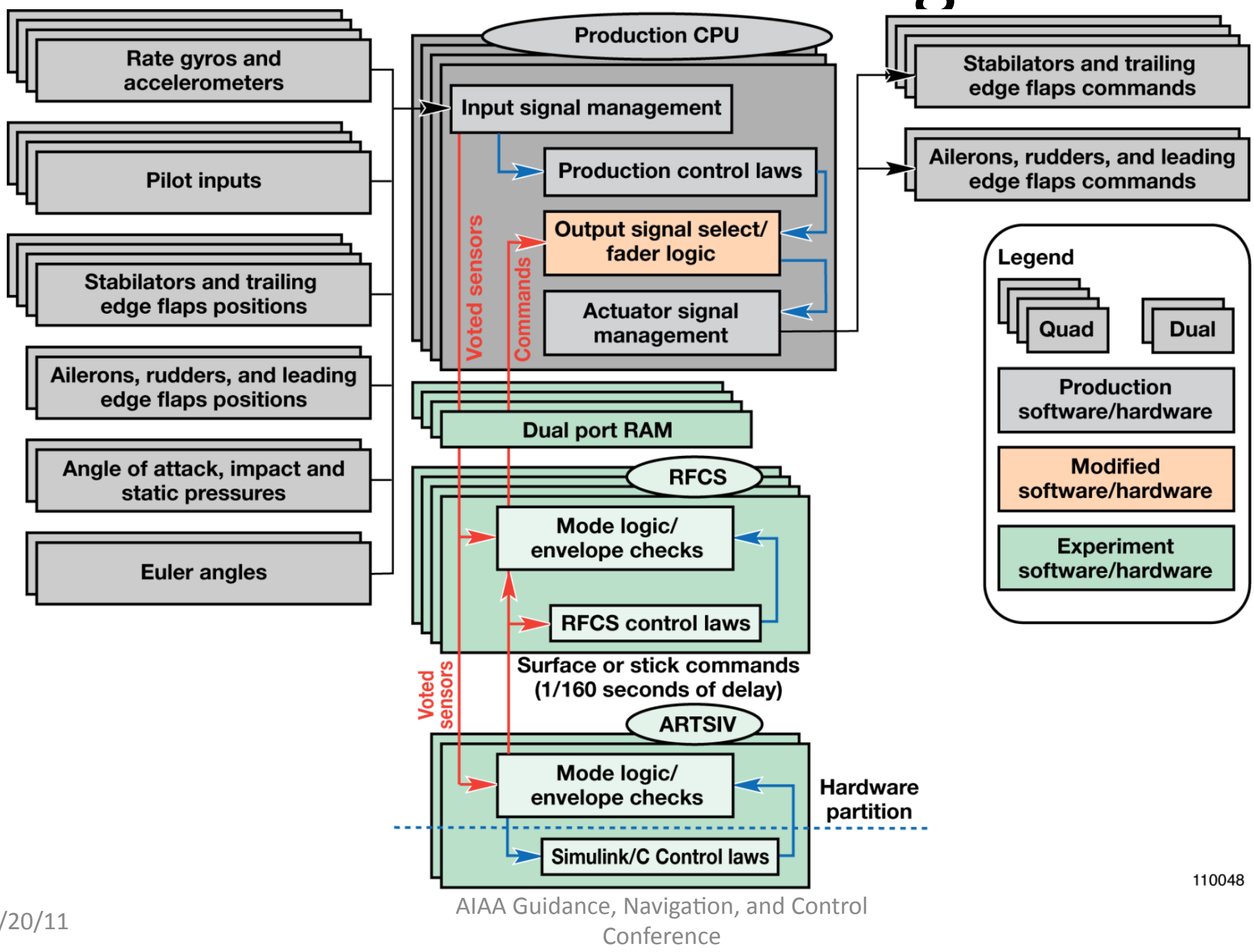




\section{FAST Envelope}

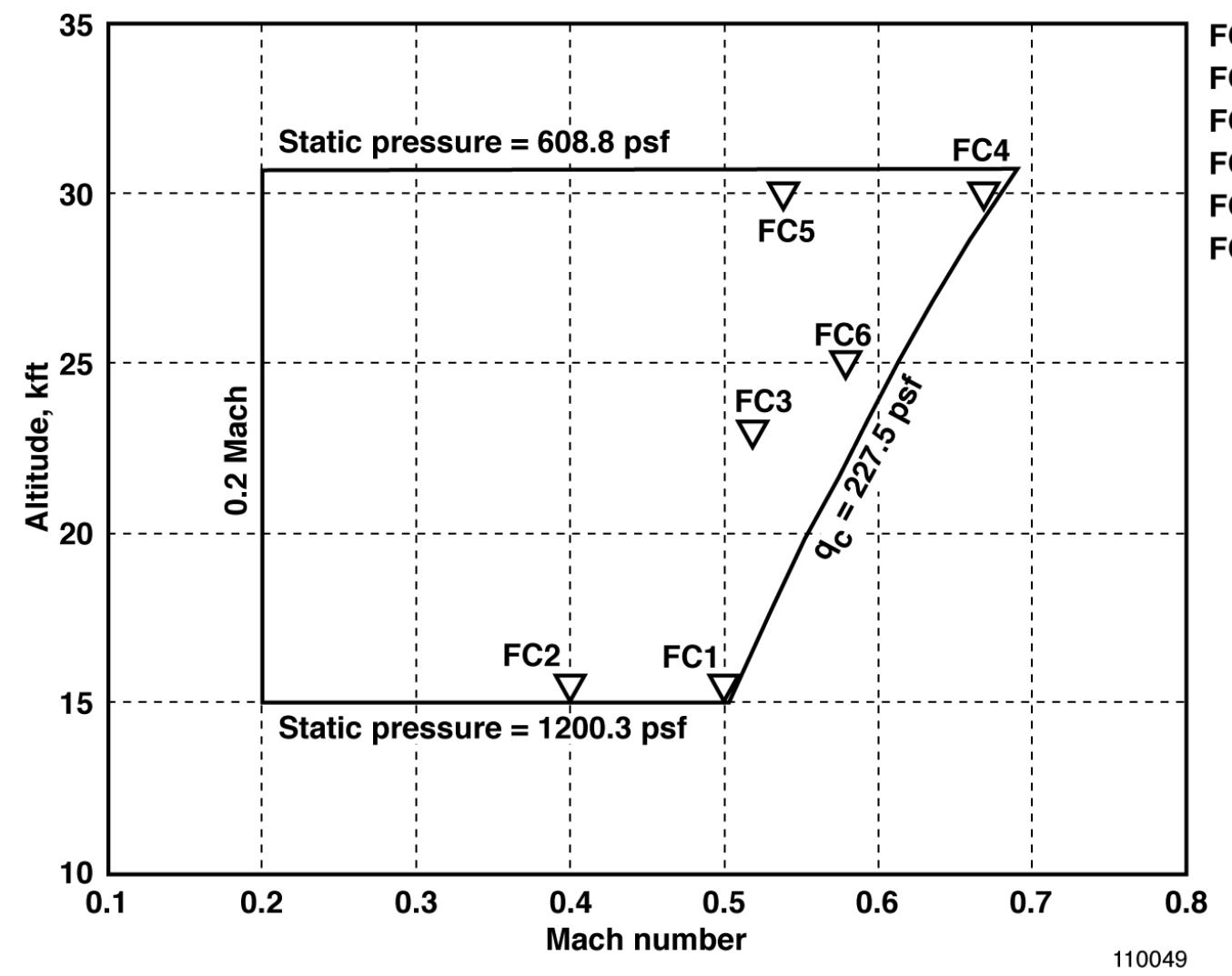

FC1: 250 KCAS, 15.5 kft FC2: 200 KCAS, $15.5 \mathrm{kft}$ FC3: $223 \mathrm{KCAS}, 23.0 \mathrm{kft}$ FC4: $250 \mathrm{KCAS}, 30.0 \mathrm{kft}$ FC5: $200 \mathrm{KCAS}, 30.0 \mathrm{kft}$ FC6: 240 KCAS, $25.0 \mathrm{kft}$ 


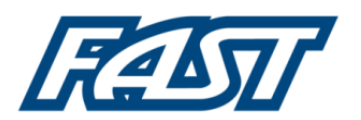

NDI Control Law Background 


\section{Control Law Overview}

- 5 Major Components

- Dynamic Inversion

- Computes surface positions to produce desired dynamics

- Aerodynamic Tables with Control Mixing

- Tabulates control surface effectiveness, and manages surface usage priorities

- Reference Models

- Compute desired vehicle dynamics from pilot commands

- PI Compensator

- Adds robustness and disturbance rejection

- Structural Filters

- Prevent undesirable ASE effects

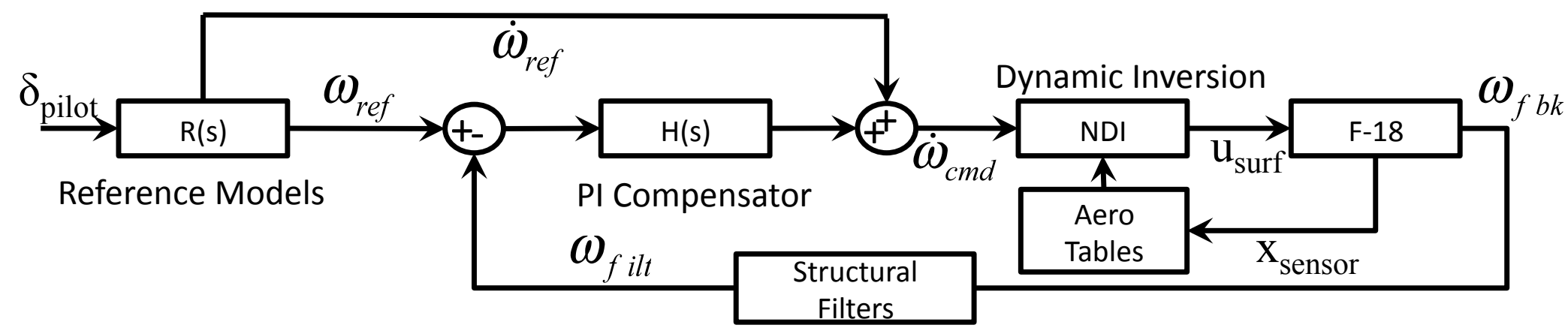




\section{Dynamic Inversion}

- Conservation of Angular Momentum Formulation

- Simple formulation that provides an architecture where the commands are body rate derivatives

- Provides structure for choosing between different trim surface positions ( $u_{\text {trim }}$ must be in the null space of $B$ )

- Mass properties assumed constant because their real time values are not available in the ARTS (no significant effect)

$$
\begin{aligned}
& \overrightarrow{H^{\varepsilon}}=I \overrightarrow{\dot{\omega}^{b}}+\overrightarrow{\omega^{b}} \times I \overrightarrow{\omega^{b}}=\overrightarrow{M^{b}}=\left[\begin{array}{l}
L \\
M \\
N
\end{array}\right]=\bar{q} S A x+\bar{q} S B\left(u-u_{\text {trim }}\right) \\
& u=w_{p}^{-1} B^{T}\left(B w_{p}^{-1} B^{T}\right)^{-1}\left[\frac{1}{\bar{q} S}\left(\frac{\overrightarrow{\omega_{c}^{b}}}{\omega^{b}} \times I \overrightarrow{\omega^{b}}\right)-A x\right]+u_{\text {trim }}
\end{aligned}
$$

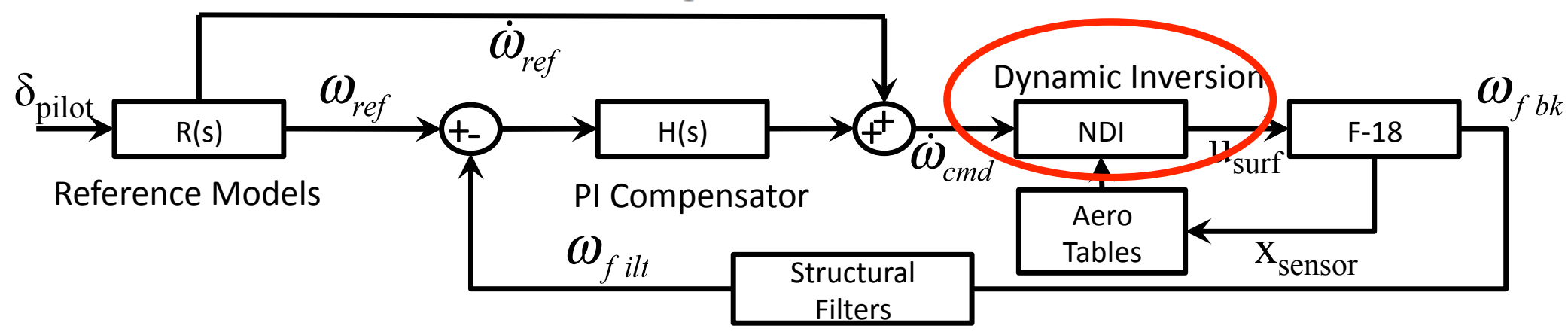




\section{Aero Tables and Mixing}

- Tabulated values assuming superposition of linear derivatives

- Current tables implemented are the AFU Navy tables in the simulation (some minor modifications to the formulation of a couple of the tables)

- Flexibility coefficients, speed brake coefficients, stores coefficients, and high mach corrections have been removed

- Sideslip lookups have not been removed; however they are currently driven with zero because there is no sideslip measurement into the ARTS (Noticeable, but acceptable effect)

- Control mixing $\left(\mathrm{w}_{\mathrm{p}}^{-1}\right)$ tailored to mimic baseline F/A-18 control mixing to a reasonable level (ailerons for roll with some differential tail and TEF, etc.)

$$
u=\overrightarrow{w_{p}^{-1} B^{T}\left(B w_{p}^{-1} B^{T}\right)^{-1}}\left[\frac{1}{\bar{q} S}\left(\overrightarrow{I \omega_{c}^{b}}+\overrightarrow{\omega^{b}} \times I \overrightarrow{\omega^{b}}\right)-A x\right]+u_{\text {trim }}
$$

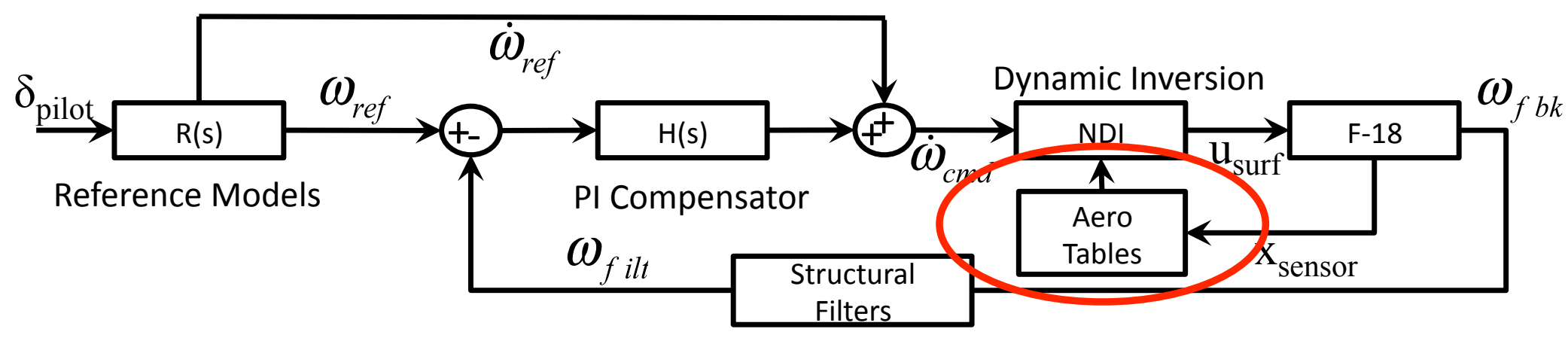




\section{Pitch Reference Model}

- Short Period approximation from MIL-STD-1797B minus the time delay compensation

- Transfer function coefficients chosen based on achieving predicted level 1 handling qualities

- Currently fixed coefficients; however these could be easily gain scheduled for wider envelopes

- Chosen over a more conventional first or second order filter for handling qualities reasons (pilots tend to give poor ratings to pure pitch rate command systems and this architecture mimics a more desirable normal acceleration command system's behavior)

- Did not result in any undesirable zero dynamics

$$
\frac{q_{r e f}}{d e p}=\frac{K_{l o n} \omega_{s p}^{2}\left(s+L_{\alpha \alpha}\right)}{s^{2}+2 \varsigma_{s p} \omega_{s p} s+\omega_{s p}^{2}} \quad \frac{\dot{q}_{r e f}}{d e p}=\frac{K_{l o n} \omega_{s s}^{2} s\left(s+L_{\alpha \alpha}\right)}{s^{2}+2 \varsigma_{s p} \omega_{s p} s+\omega_{s p}^{2}}
$$

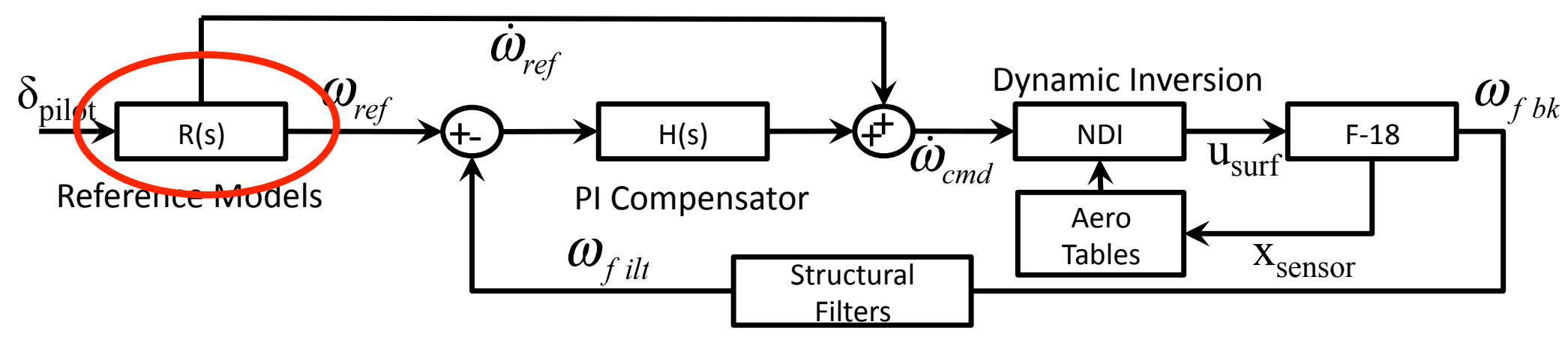




\section{Roll Reference Model}

- Roll mode approximation from MIL-STD-1797B minus the time delay compensation

- Roll commands are about the stability axis to prevent the undesirable bunting behavior for body axis rolls (not a complete solution)

- Second order stick shaping is added to reduce the sensitivity to small roll stick commands but preserve the ability to command large roll rates for large stick deflections

- Roll gain shaping scheduled with angle of attack to dial back the roll gain for moderate to high angles of attack (7-12 degrees) to account for insufficient yaw control power to coordinate these rolls

- Did not result in undesirable zero dynamics

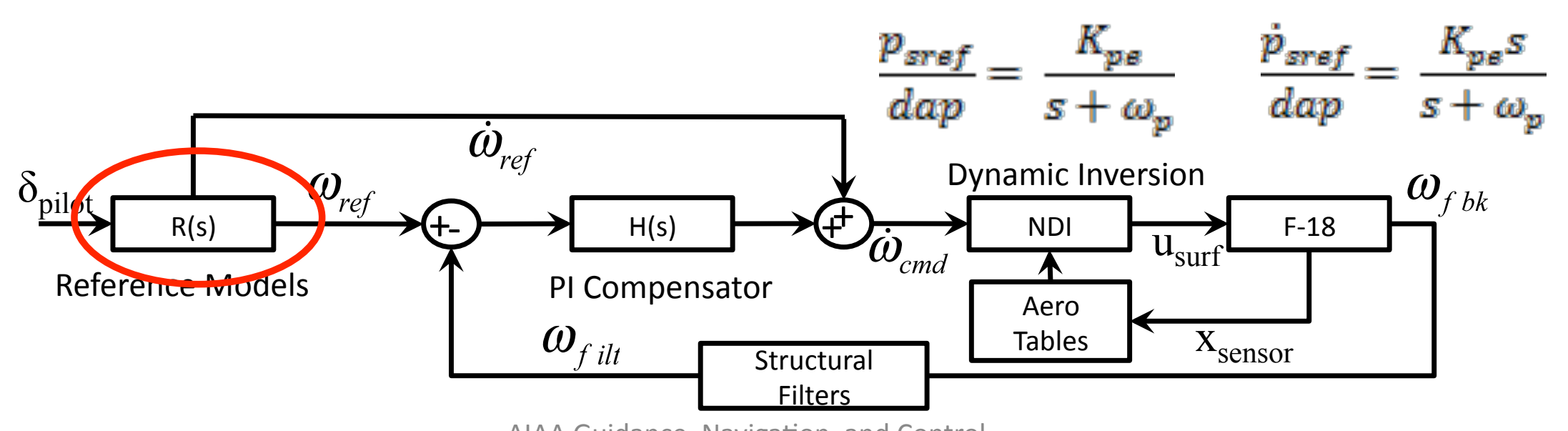




\section{Yaw Reference Model}

- Stability axis yaw rate command used to coordinate stability axis roll rate command $(\dot{\beta} \approx 0)$ and generate pedal commanded lateral acceleration

- First order filter used to smooth and differentiate the stability axis yaw rate command

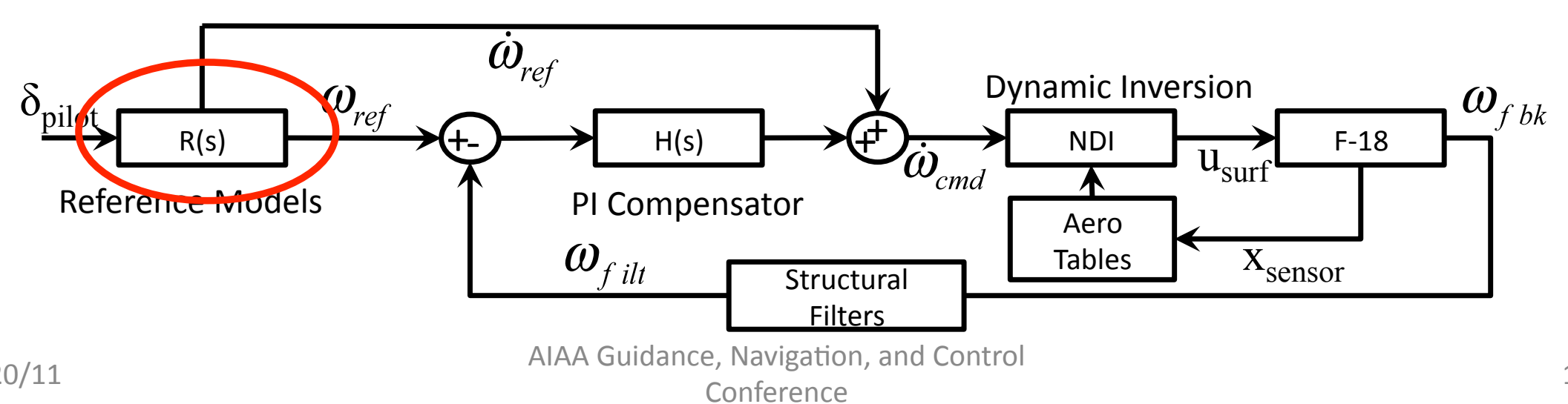




\section{Proportional-Plus-Integral Compensator}

- Provide robustness to the overall design and help account for modeling inaccuracies in the Dynamic Inverse

- Compensators tuned primarily for disturbance rejection

- Overall closed loop system should not be overly sensitive to disturbances as this causes undesired oscillations

- An additional MRAC requirement is that that the gains of the PI compensator be chosen such that the closed loop system response to disturbances has the same poles as the reference model for that particular axis

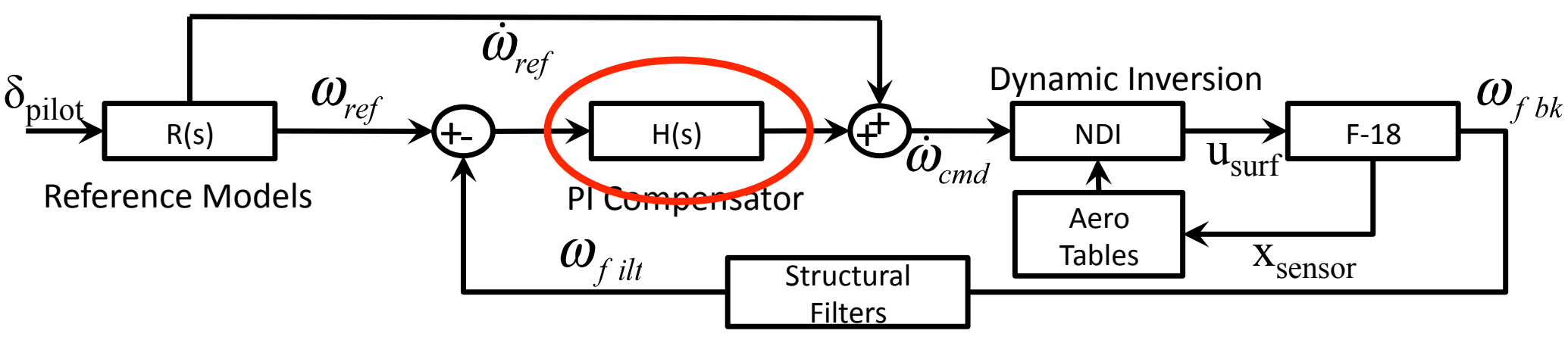


F每区

\section{Control Law Evaluation}




\section{Types of Analyses}

- Linear stability margins (not presented)

- Closed loop tracking performance

- Handling qualities metrics

- Low Order Equivalent Systems (LOES)

- Neal-Smith Criteria

- Bandwidth Criteria

- Piloted Simulation Tests 


\section{Reference Model Tracking}

- Tracking the design reference model serves two purposes

- Tracking a well designed reference model (discussed previously) should yield the handling qualities predicted for that reference model

- MIL Specifications exist for the standard aircraft modes

- The reference model command is used to generate the tracking error used to "train" the adaptive control laws

- Keeping the error small for non-failed configurations keeps an adaptive controller from adapting when it is not required to do so

- Good tracking defined: the difference between the closed loop response and the reference model are indiscernible to a pilot.

- Maximum Unnoticeable Added Dynamics (MUAD) envelopes in the frequency domain

- Compensation required for actuator dynamics

- Actuators (or any other delay source) tend to increase the apparent natural frequency and reduce the apparent damping ratio for the closed loop system when compared to the commanded reference model due to their rate limits and bandwidth limitations 


\section{Pitch Reference Model Tracking}

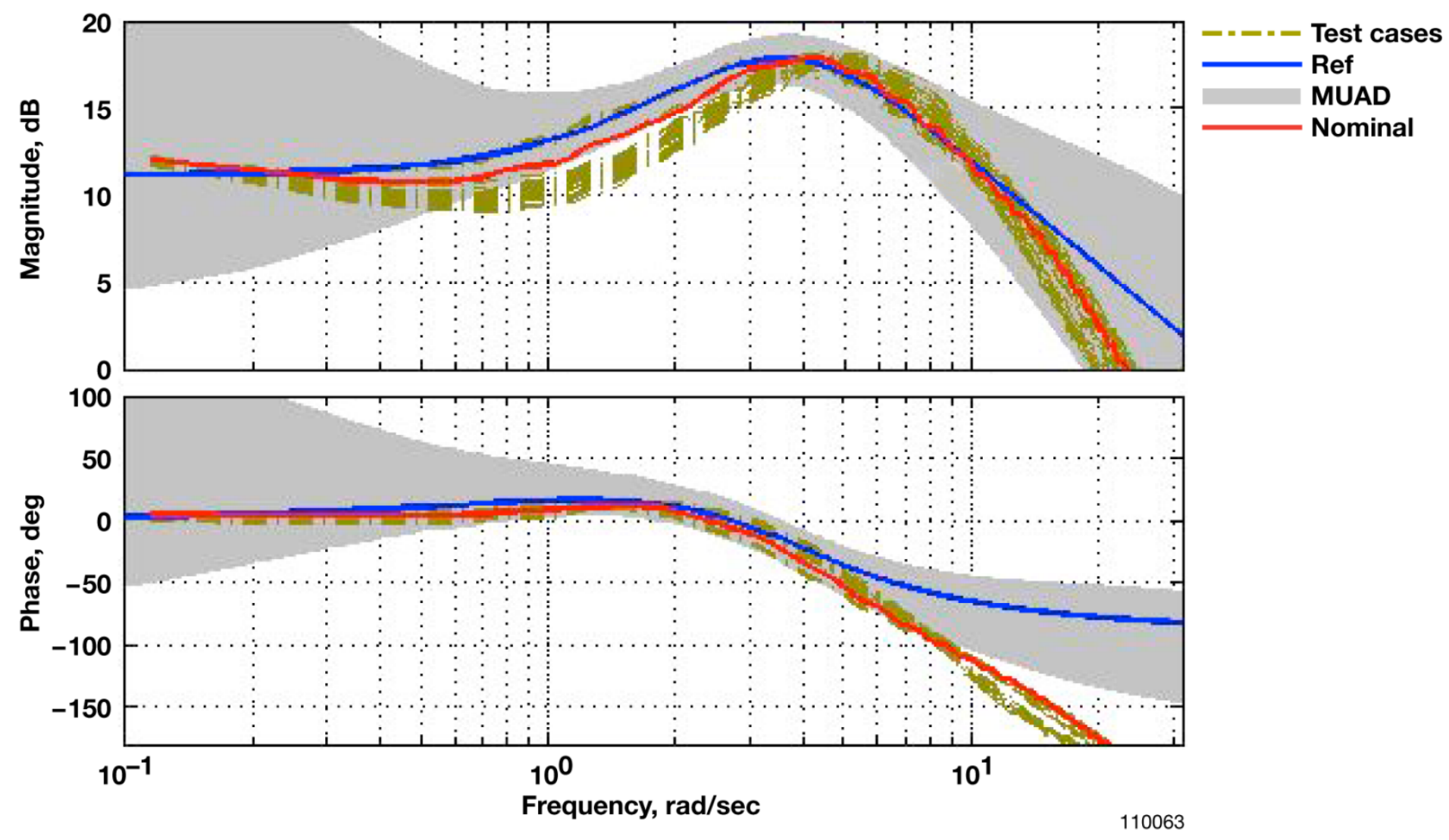




\section{Longitudinal HQ General Comments}

- Many metrics exist due to the important nature of the this axis

- Most modern control laws are a mix between pitch rate, angle of attack, and normal acceleration command systems

- The current NDI is a pure pitch rate command system

- Chosen for simplicity of integration with MRAC and other follow-on research control laws

- Proper selection of a pitch rate reference model alleviates most of the undesirable aspects of a pure pitch rate architecture.

- Requires gain scheduling with flight condition

- Does not address some undesirable roll axis behavior caused by pitch rate command architecture 


\section{Handling Qualities (LOES)}
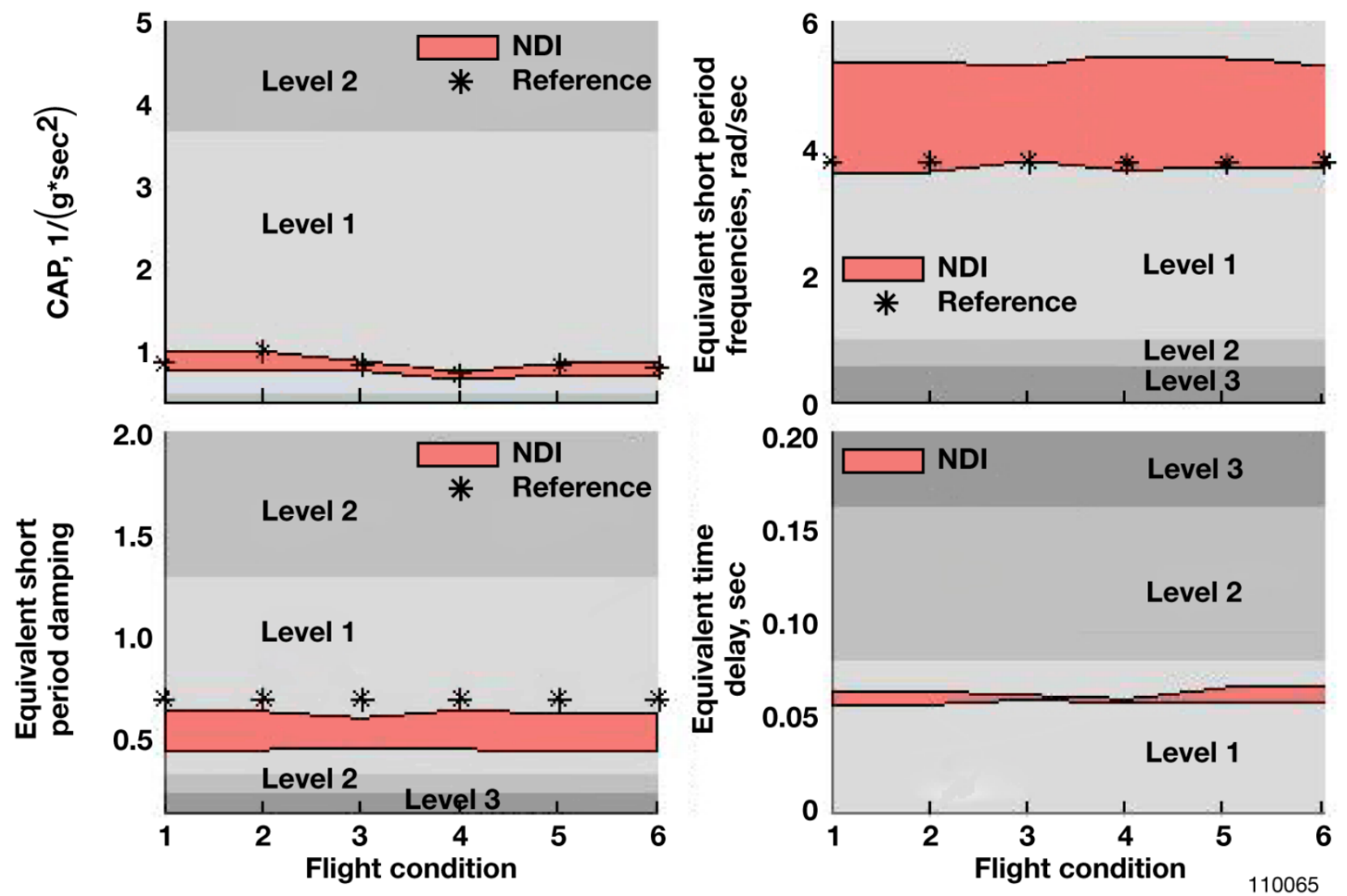

$$
\frac{\mathrm{q}}{\mathrm{F}_{\mathrm{s}} \text { or } \delta_{\mathrm{s}}}=\frac{\mathrm{K}_{\mathrm{q}_{\mathrm{e}}}\left(\mathrm{s}+\frac{1}{\mathrm{~T}_{\theta_{2}}}\right) \mathrm{e}^{-\tau_{\theta_{\mathrm{e}}} \mathrm{s}}}{\left(\mathrm{s}^{2}+2 \zeta_{\mathrm{sp}_{\mathrm{e}}} \omega_{\mathrm{sp}_{\mathrm{e}}} \mathrm{s}+\omega_{\mathrm{sp}_{\mathrm{e}}}^{2}\right)}
$$




\section{Handling Qualities (Neal-Smith)}

- Metric background

- Pilot oriented tool

- Based on a pilot/compensator model

- Commonly used to predict cliffs due to delays

- Tends to be stringent

- As applied to NDI

- Fixed pilot time delay of $0.2 \mathrm{sec}$

- Based on results generated with production control law

- Varied pilot bandwidth from 1.5-5.5 rad/sec

- Spans the normal 2-3 rad/sec generally considered as accurate for most pilots

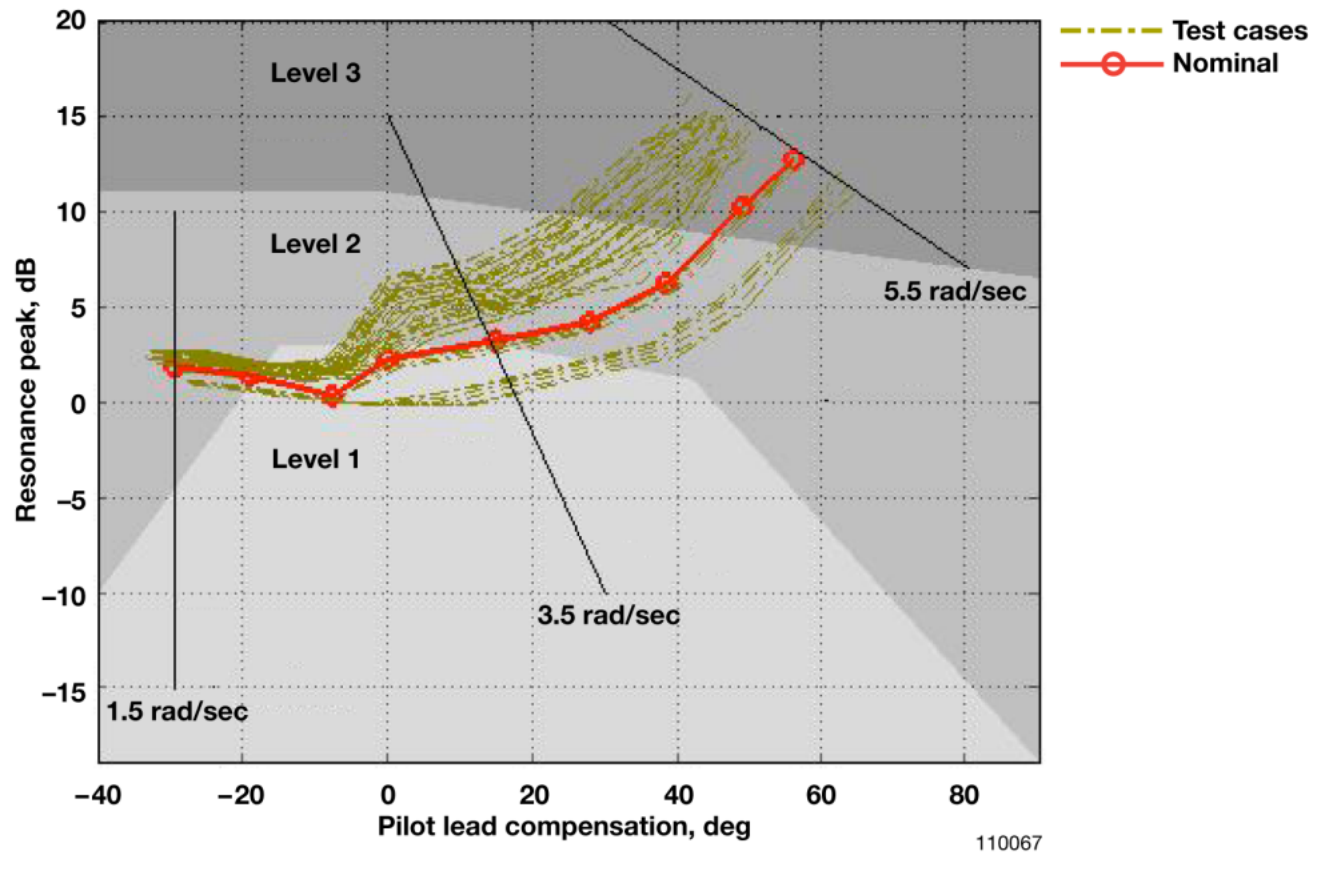




\section{Handling Qualities (Bandwidth)}

- Metric Background

- Pilot oriented

- Treats pilot like a simple gain

- Has been shown to be useful in predicting $\mathrm{PIO}$

- Application to NDI

- No modification from published

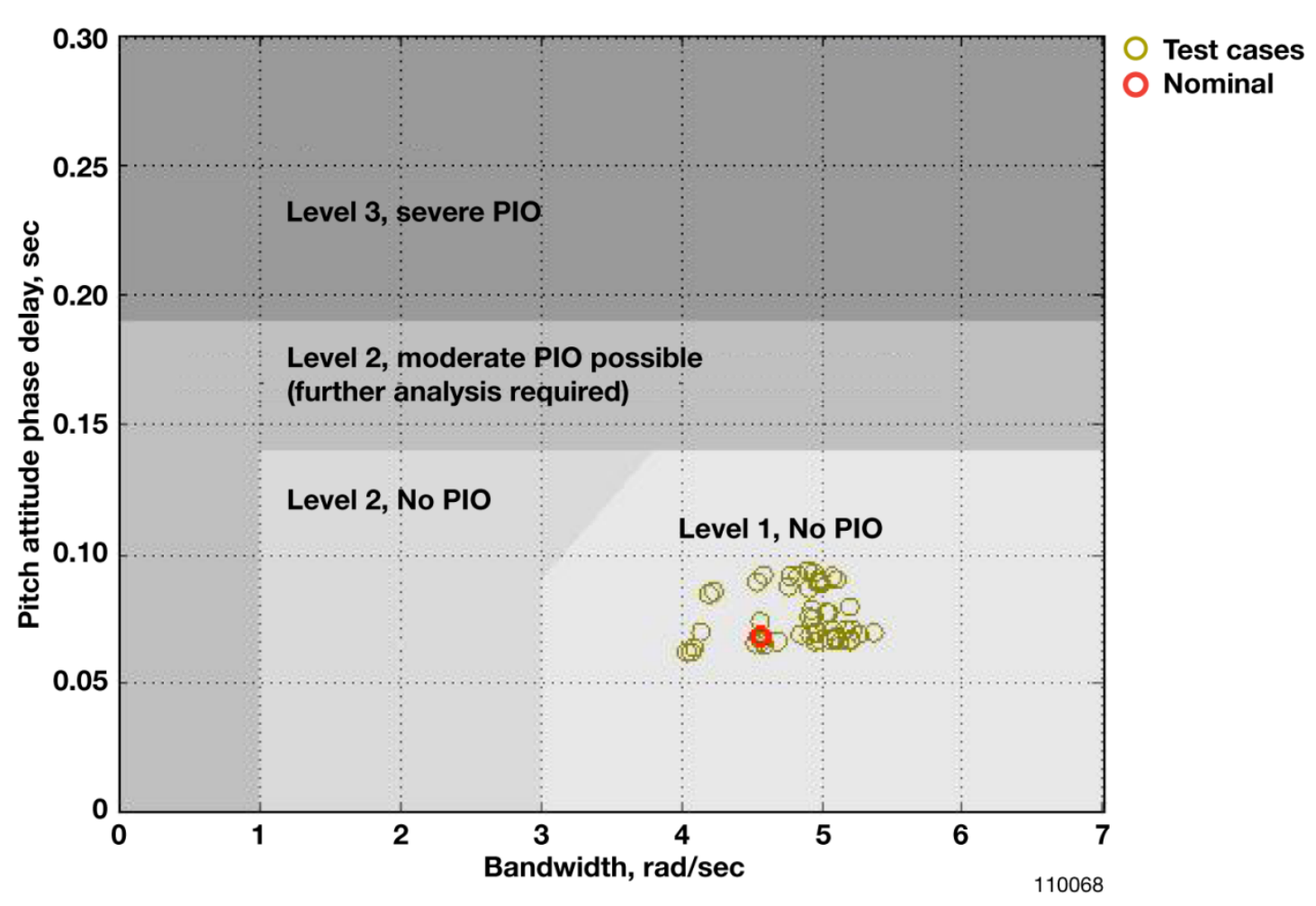
techniques 


\section{Pilot Comments Simulation Testing}

- Flight condition 6 (25,000ft, Mach 0.58)

- Overall feels like a normal F-18

- Stick forces slightly heavier in pitch

- Good damping in all axes

- Pitch captures exhibit additional overshoots

- Related to prominence of resonant peak in the pitch reference model

- Roll captures show no noticeable difference

- SHSS are flatter (require less bank to maintain heading)

- Flight condition $4(30,000 \mathrm{ft}$, Mach 0.67)

- Very similar to FC6

- Flight condition $2(15,500 \mathrm{ft}$, Mach 0.4)

- Similar to FC6

- Flight condition $5(30,00 \mathrm{ft}$, Mach 0.54)

- Very similar to FC2 


\section{Conclusions}

- NDI architecture presented has been shown to be sufficiently robust and to produce predicted Level 1 handling qualities

- Angular momentum formulation, reference model selection, aerodynamic formulation, control weighting, and $\mathrm{PI}$ compensator tuning

- NDI provides a good baseline control law upon which advanced control elements (adaptive, structural control) can be built and taken to flight quickly 


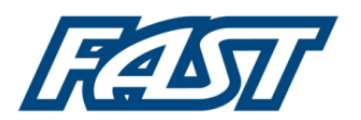

\section{Questions}




\section{Roll Reference Model Tracking}
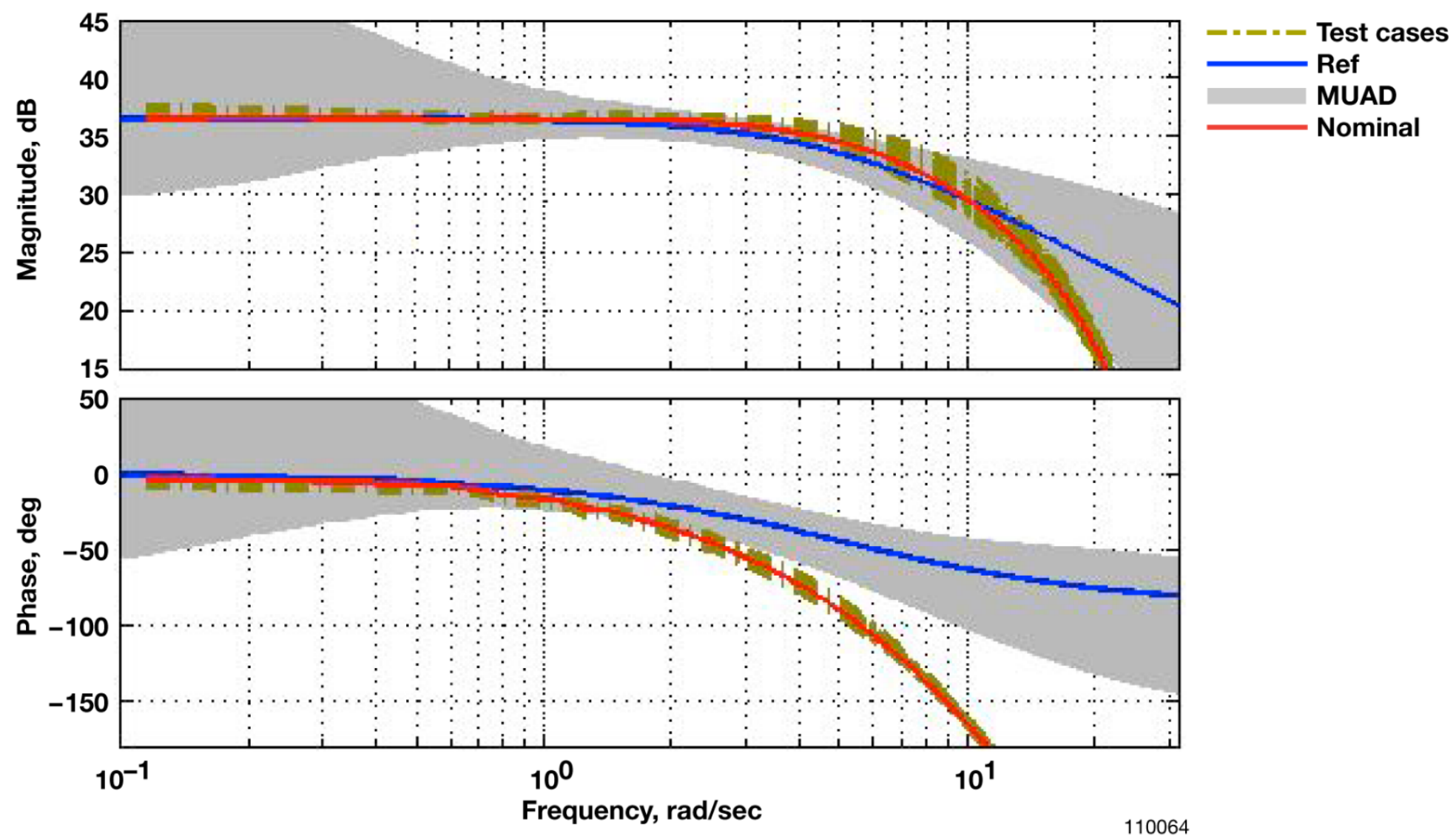


\section{Pitch Open Loop}

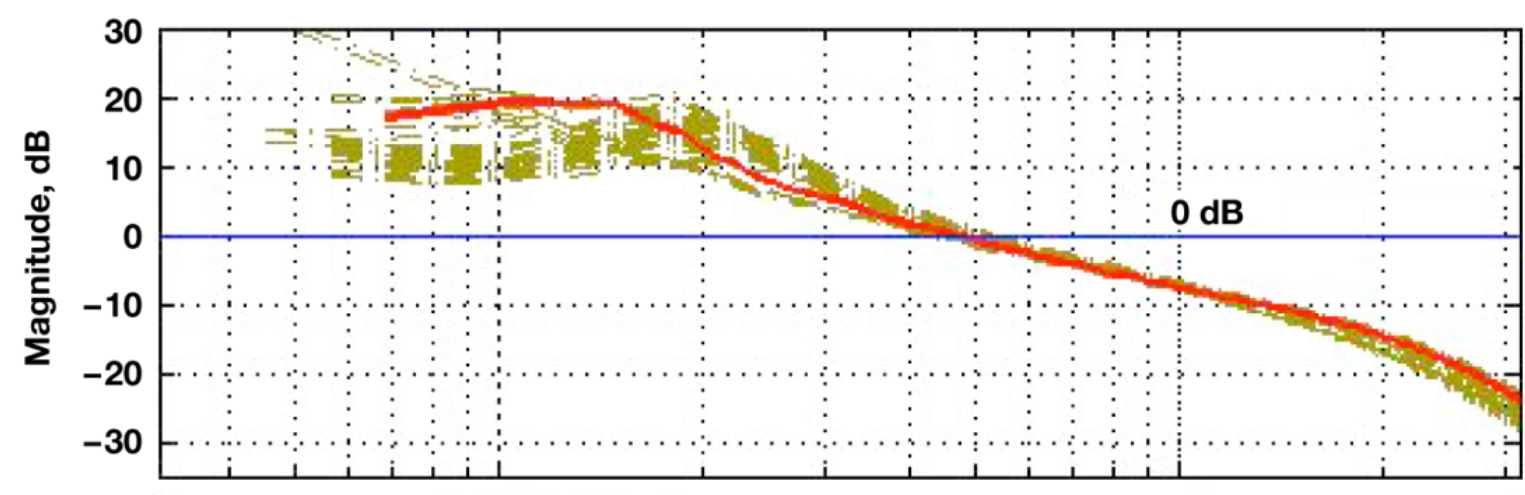

$-=---$ Test cases Nominal

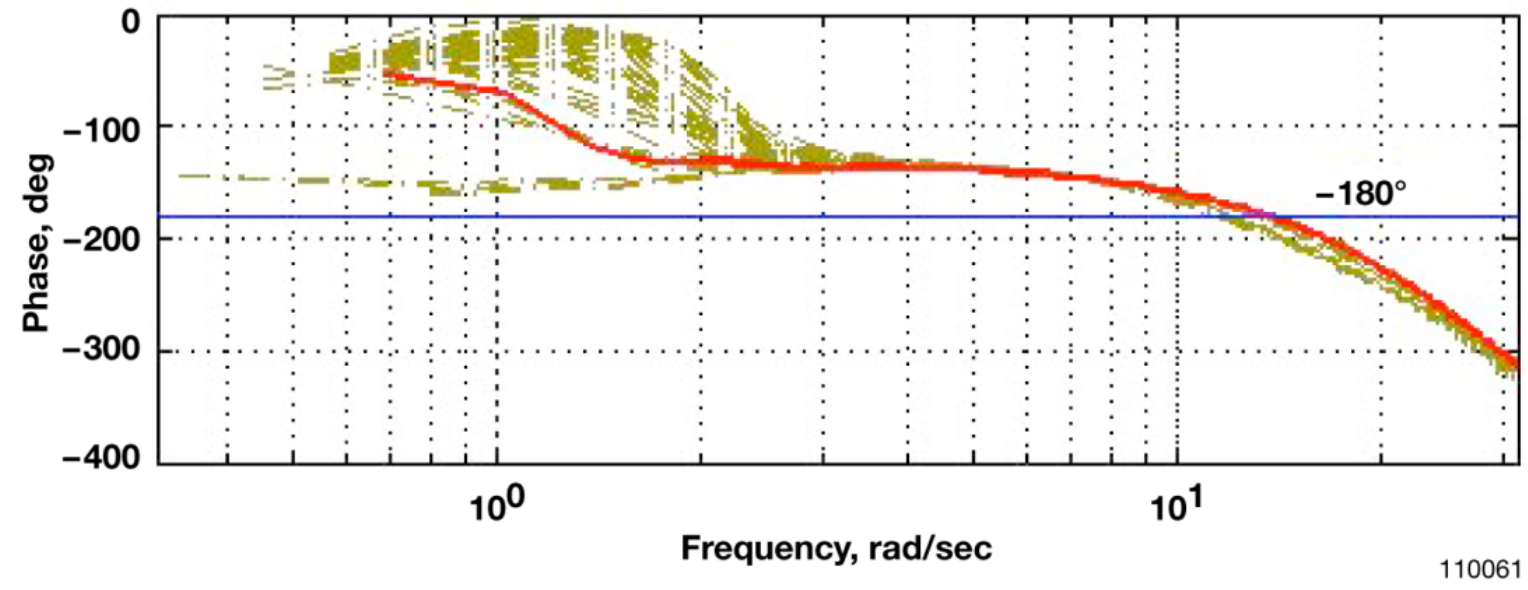




\section{Pitch Margins}
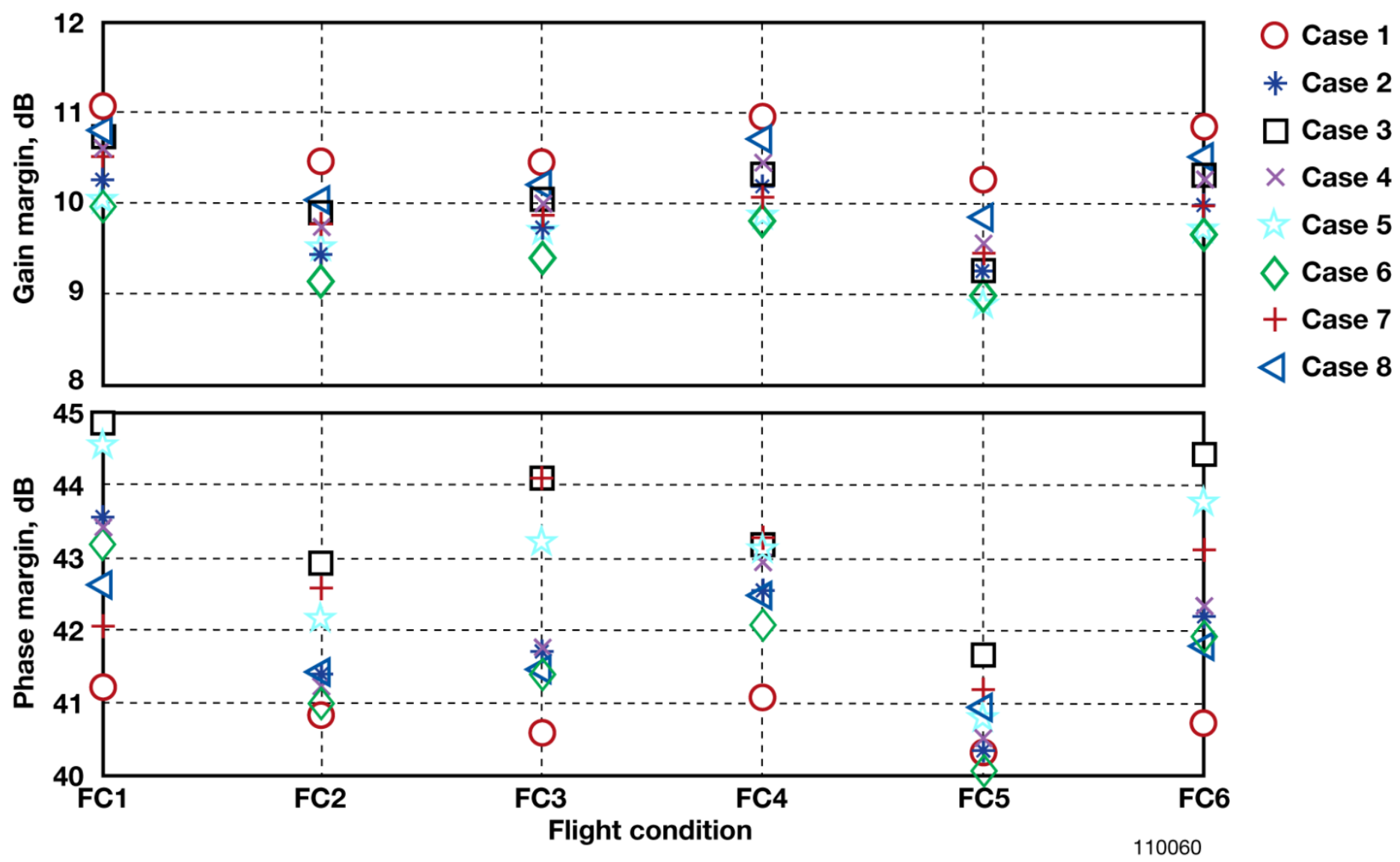


\section{Roll Margins}
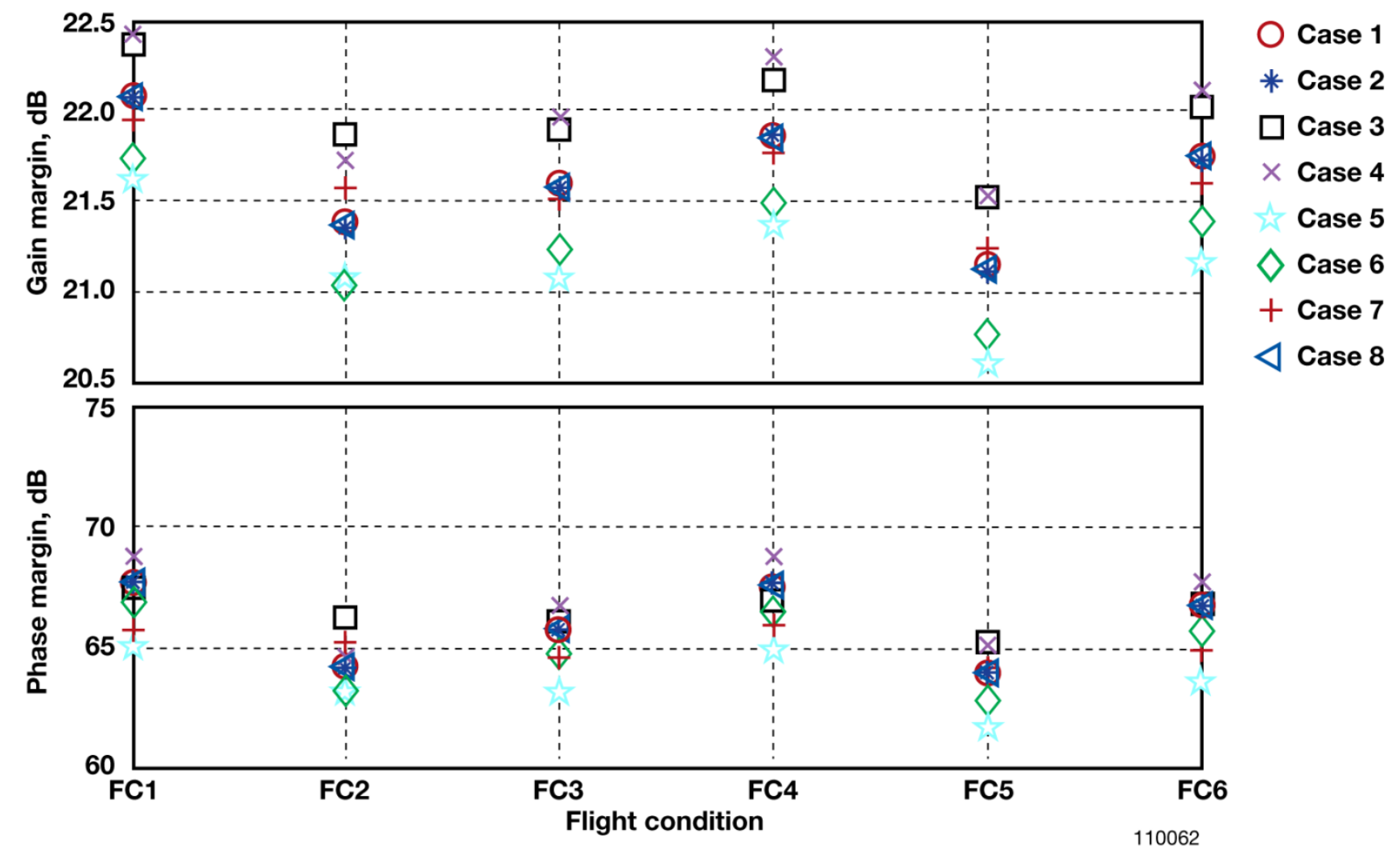


\section{Roll HQ General Comments}

- Pilots tend to be more flexible with regard to roll axis behavior than to pitch

- Roll metrics sparse, and containing fewer verification studies

- Primary driver for the NDI design was control harmony with the pitch axis for loaded roll maneuvers

- Roll and yaw axes are coupled and therefore yaw handling qualities affect the roll handling qualities

- Yaw designed to coordinate stability axis rolls

- Stability axis chosen due to the effect that high rate body axis rolls has on the pitch axis 


\section{Yaw HQ Comments}

- Number and substantiation of yaw metrics even more sparse than roll

- Found that minimizing the yaw axis' effects on the roll behavior was more important that matching the Mil standard reference model form

- Faster and better coordination trumped having a familiar dutch-roll mode that satisfied the mil standard

- The suggested requirements in 1797 were found to be far too loose to be useful for this design 